This item was submitted to Loughborough's Research Repository by the author.

Items in Figshare are protected by copyright, with all rights reserved, unless otherwise indicated.

\title{
Mixed-mode delamination in layered isotropic and laminated composite beam structures
}

PLEASE CITE THE PUBLISHED VERSION

http://www.appleacademicpress.com/

\section{PUBLISHER}

(C) Apple Academic Press

\section{VERSION}

AM (Accepted Manuscript)

\section{PUBLISHER STATEMENT}

This work is made available according to the conditions of the Creative Commons Attribution-NonCommercialNoDerivatives 4.0 International (CC BY-NC-ND 4.0) licence. Full details of this licence are available at: https://creativecommons.org/licenses/by-nc-nd/4.0/

\section{LICENCE}

CC BY-NC-ND 4.0

\section{REPOSITORY RECORD}

Harvey, Christopher M., Paul Cunningham, and Simon Wang. 2015. "Mixed-mode Delamination in Layered Isotropic and Laminated Composite Beam Structures”. figshare. https://hdl.handle.net/2134/18855. 


\title{
Mixed-mode delamination in layered isotropic and laminated composite beam structures
}

\author{
Christopher Martin Harvey, Paul Cunningham, and Simon Wang
}

\section{Contents}

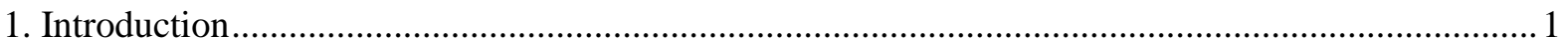

2. Clamped-clamped isotropic beams ................................................................................... 4

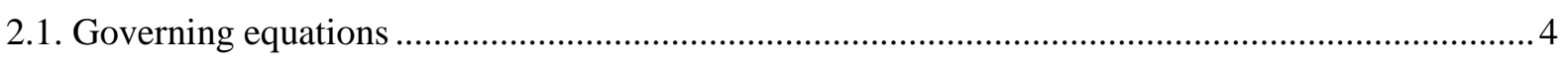

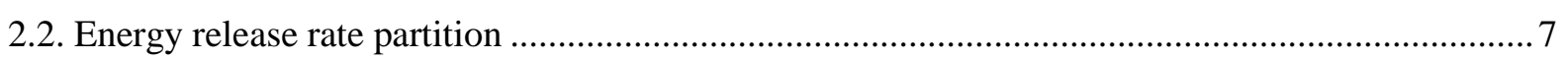

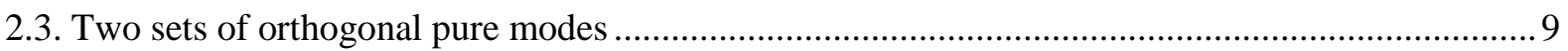

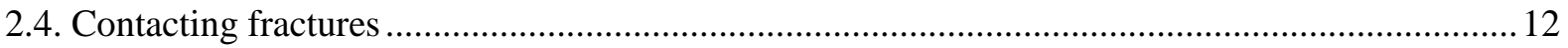

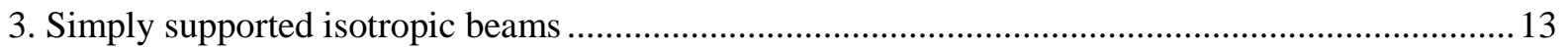

4. Clamped-clamped laminated composite beams ................................................................................ 14

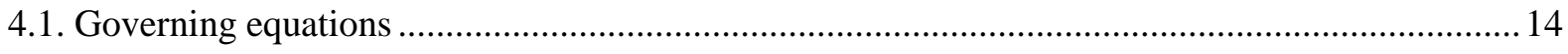

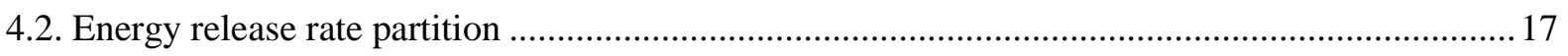

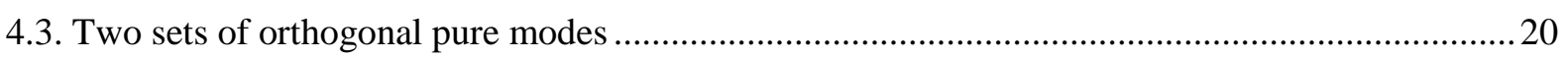

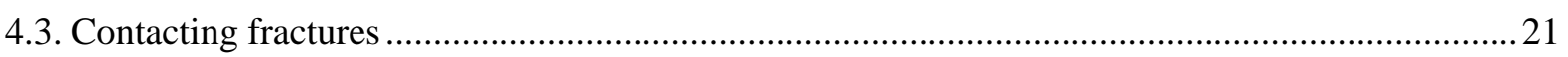

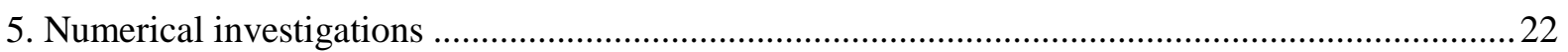

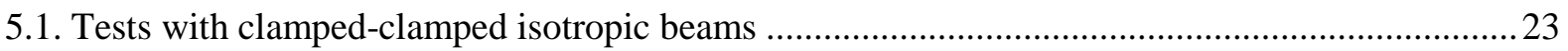

5.2. Tests with clamped-clamped laminated composite beams ..............................................................24

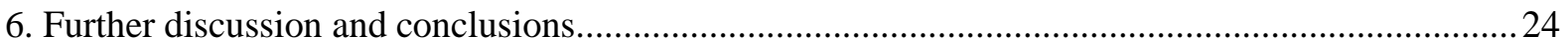

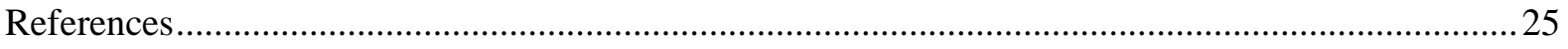

\begin{abstract}
Completely analytical theories are presented for calculating the total energy release rate (ERR) in a mixed-mode delamination in layered isotropic and laminated composite straight beam structures and for partitioning it into opening mode I and shearing mode II components. The theories are developed within the contexts of both the Euler and Timoshenko beam theories. The theories are extensively verified against numerical simulations using the finite element method. The developed theories provide a valuable means for the design of such beam structures against delamination.
\end{abstract}

\section{Introduction}

Layered isotropic and laminated composite straight beams are commonly used in many different engineering structures, such as aircraft, buildings and bridges, etc. Delamination is a major concern in these applications, for example, a commonly-used method to repair or strengthen a concrete beam in 
civil engineering is to bond either a metal plate or a carbon-fiber-reinforced laminate onto it. The fracture toughness against debonding at the interface is a crucial design parameter. In general, debonding is a mixed-mode fracture, that is, it consists of both mode I opening and mode II shearing. The toughness depends on the proportions of these two individual fracture modes. Therefore, it is imperative to partition the total energy release rate (ERR) of a mixed-mode fracture into its mode I and II components which govern the fracture toughness or the fracture propagation criterion.

Some of the earliest analytical work on the topic of one-dimensional fracture-that is, fracture which propagates in one direction with mode I and mode II components only-was reported by Williams (1), who made some significant contributions to the understanding for isotropic double cantilever beams (DCBs). A semi-analytical partition theory was given by Schapery and Davidson (2), which was also for isotropic DCBs and based on Euler beam theory. They were not able to give Williams's (1) pair of pure modes and claimed that Euler beam theory doesn’t provide quite enough information to obtain a decomposition of energy release rate into opening and shearing mode components. They therefore used the finite element method (FEM) to solve the two-dimensional continuum problem around the crack tip in order to partition the energy release rate. Suo and Hutchinson (3-5) used a similar approach to Schapery and Davidson (2), but instead of using the FEM, they used integral equation methods to obtain a two-dimensional linear elasticity solution for the crack tip region. The resulting partition theory is analytical except for one parameter, which is determined numerically. Schapery and Davidson's (2) and Suo and Hutchinson's (3-5) partition theories generally give different partitions to William's (1) partition theory. Zou et al. (6) derived a completely analytical partition theory for isotropic DCBs based on Timoshenko beam theory. Bruno and Greco (7) obtained the same partition but for Euler beams instead of Timoshenko beams. Luo and Tong (8) derived the same partition theory as Bruno and Greco (7), also for Euler beams, but by a different method. None of the work by Zou et al. (6), Bruno and Greco (7) and Luo and Tong (8) is in agreement with Williams’s (1), Schapery and Davidson’s (2), or Suo and Hutchinson’s (3) partition theories.

Recently, based on a fundamental physical understanding and a complete mechanical representation of the problem, a powerful mathematical methodology has been created by the authors to partition the total ERR. Several challenging fracture problems have been solved analytically. The research results have been reported in a series of publications (9-18). The authors' mixed-mode partition theory based on classical laminate theory has been shown $(11,18)$ to agree very well with the test data obtained from a series of experimental studies by different research groups (19-23). Also, the authors' latest work $(26,27)$ shows that authors' mixed-mode partition theory based on first-order shear-deformable laminate theory plays a key role in the development of a local mixed-mode partition theory between two dissimilar elastic materials. 
The major aim of the present study is to extend the previous work (9-18) to develop analytical theories to calculate the total ERR and its mode I and II partitions for a mixed-mode delamination in layered isotropic and laminated composite straight beams under various loading conditions and boundary conditions. The work provides a valuable means for the design of such beam structures against delamination.

The structure of this paper is as follows: Sections 2 and 3 develop analytical theories for layered isotropic beams; Section 4 develops analytical theories for laminated composite beams; numerical tests are presented in Section 5; and further discussions and conclusions are made in Section 6.

\section{Nomenclature}

$a$

$A_{1}, A_{2}, A$

$b$

$B_{1}, B_{2}, B$

$D_{1}, D_{2}, D$

$D_{o p}, D_{s h}$

$E$

$F_{n B}, F_{s B}$

$F_{n B \theta_{1}}$

$F_{n B P}$

$G, G_{I}, G_{I I}$

$G_{P}$

$G_{x z}$

$G_{\theta_{i}}, G_{\beta_{i}}$

$h_{1}, h_{2}, h$

$H_{1}, H_{2}, H$

$I_{1}, I_{2}, I$

$L_{1}, L_{2}$

$M_{1}, M_{2}$

$M_{1 B_{i}}, M_{2 B_{i}}, M_{B_{i}}$ length of fracture

extensional stiffness of upper, lower and intact beams

beam width

coupling stiffness of upper, lower and intact beams

bending stiffness of upper, lower and intact beams

crack tip relative opening and shearing displacements

Young's modulus

crack tip normal and shear forces

crack tip opening force due to mode $\varphi_{\theta_{1}}$

crack tip opening force due to shearing

total, mode I and mode II energy release rates

mode I energy release rate due to shearing

shear modulus

energy release rates due to mode $\varphi_{\theta_{i}}$ and mode $\varphi_{\beta_{i}}$

thicknesses of upper, lower and intact beams

out-of-plane shearing stiffness of upper, lower and intact beams

second moments of area of upper, lower and intact beams

length of left and right intact parts of beam

bending loads acting on upper and lower beams

bending moments on upper, lower and intact beams at crack tip $i$ 


\begin{tabular}{|c|c|}
\hline$N_{1}, N_{2}$ & axial loads acting on upper and lower beams \\
\hline$N_{1 B_{i}}, N_{2 B_{i}}, N_{B_{i}}$ & axial forces on upper, lower and intact beams at crack tip $i$ \\
\hline$P_{c}$ & point contact force \\
\hline$P_{1}, P_{2}$ & shear loads acting on upper and lower beams \\
\hline$P_{1 B_{i}}, P_{2 B_{i}}, P_{B_{i}}$ & crack tip shear forces on upper, lower and intact beams at crack tip $i$ \\
\hline$u_{1}, u_{2}, u_{3}, u_{4}$ & axial displacements of the upper, lower, left and right beams \\
\hline$w_{1}, w_{2}, w_{3}, w_{4}$ & deflections of the upper, lower, left and right beams \\
\hline$x_{p_{1}}, x_{p_{2}}$ & distance from left crack tip to loading location on upper and lower beams \\
\hline$\alpha_{\varphi_{i}}$ & mixed mode partition coefficient for mode $\varphi_{i}$ \\
\hline$\beta_{i}, \beta_{i}^{\prime}$ & the two pure mode II 'crack tip modes' from the ith set \\
\hline$\beta_{F}, \beta_{F}^{\prime}$ & the two pure mode II ' $\mathrm{F}$ modes' \\
\hline$\gamma$ & thickness ratio $h_{2} / h_{1}$ \\
\hline$\Delta G_{\varphi_{i} \varphi_{j}}$ & energy release rate interaction between modes $\varphi_{i}$ and $\varphi_{j}$ \\
\hline$\theta_{i}, \theta_{i}^{\prime}$ & the two pure mode I 'crack tip modes' from the $i$ th set \\
\hline$\theta_{F}, \theta_{F}^{\prime}$ & the two pure mode I 'F modes' \\
\hline$\kappa^{2}$ & shear correction factor \\
\hline$\varphi_{\theta_{i}}, \varphi_{\beta_{i}}$ & mode vectors for the $i$ th mode I and the $i$ th mode II \\
\hline$\psi_{1}, \psi_{2}, \psi_{3}, \psi_{4}$ & rotations of normals to mid-surface of the upper, lower, left and right bear \\
\hline
\end{tabular}

\section{Clamped-clamped isotropic beams}

\subsection{Governing equations}

A general clamped-clamped beam with a fracture is shown in Figure 1.1. Contact between the upper and lower beams is not treated initially. With reference to Figure $1.1 \mathrm{~b}$ and using the constitutive relation from Timoshenko beam theory for isotropic materials, the following are easily derived:

$$
\psi_{1,2}=\frac{1}{E I_{1,2}}\left(M_{1,2 B_{1}} x-\frac{P_{1,2 B_{1}} x^{2}}{2}-M_{1,2}\left\langle x-x_{p_{1,2}}\right\rangle+\frac{P_{1,2}}{2}\left\langle x-x_{p_{1,2}}\right\rangle^{2}\right)+\psi_{1,2 B_{1}}
$$




$$
\begin{aligned}
& w_{1,2}=\frac{1}{E I_{1,2}}\left(\frac{M_{1,2 B_{1}} x^{2}}{2}-\frac{P_{1,2 B_{1}} x^{3}}{6}-\frac{M_{1,2}}{2}\left\langle x-x_{p_{1,2}}\right\rangle^{2}+\frac{P_{1,2}}{6}\left\langle x-x_{p_{1,2}}\right\rangle^{3}\right) \\
& +\psi_{1,2 B_{1}} x+w_{1,2 B_{1}}+\frac{1}{b h_{1,2} G_{x z} \kappa^{2}}\left(P_{1,2 B_{1}} x-P_{1,2}\left\langle x-x_{p_{1,2}}\right\rangle\right) \\
& \psi_{3}=\frac{1}{E I}\left(\frac{R_{A} x^{2}}{2}-M_{A} x\right) \text { and } w_{3}=\frac{1}{E I}\left(\frac{R_{A} x^{3}}{6}-\frac{M_{A} x^{2}}{2}\right)+\frac{P_{B_{1}} x}{b h G_{x z} \kappa^{2}} \\
& \psi_{4}=\frac{1}{E I}\left(M_{B_{2}} x-M_{B_{2}} L_{2}-\frac{P_{B_{2}} x^{2}}{2}+\frac{P_{B_{2}} L_{2}^{2}}{2}\right) \\
& w_{4}=\frac{1}{E I}\left(\frac{M_{B_{2}} x^{2}}{2}-M_{B_{2}} L_{2} x+\frac{M_{B_{2}} L_{2}{ }^{2}}{2}-\frac{P_{B_{2}} x^{3}}{6}+\frac{P_{B_{2}} L_{2}{ }^{2} x}{2}-\frac{P_{B_{2}} L_{2}{ }^{3}}{3}\right)+\frac{P_{B_{2}} x}{b h G_{x z} \kappa^{2}}
\end{aligned}
$$

Subscripts 1 and 2 refer to the upper and lower beams respectively. Subscripts 3 and 4 refer to the left- and right-hand intact laminates. The left crack tip is at location $B_{1}$. The right crack tip is at location $\mathrm{B}_{2}$. In these equations, the origin of $x$ is at $\mathrm{B}_{1}$ and to the right for beams 1 and 2; for beams 3 and 4 it is at the respective left-hand sides and to the right (as shown in Figure 1.1a); $w$ is the upward deflection; the rotations, $d w / d x$ and $\psi$ are anticlockwise. The angle brackets are Macaulay brackets, denoting the ramp function. The axial displacements of the beams are

$$
\begin{gathered}
u_{1,2}=\left(N_{1,2 B_{1}} x-N_{1,2}\left\langle x-x_{p_{1,2}}\right\rangle\right) /\left(b E h_{1,2}\right)+u_{1,2 B_{1}} \\
u_{3}=N_{B_{1}} x /(b E h) \quad, \quad u_{4}=\left(N_{B_{2}} x-N_{B_{2}} L_{2}\right) /(b E h)
\end{gathered}
$$

Equilibrium can be used to describe all the forces in Figure 1.1b in terms of the six left crack tip forces $M_{1,2 B_{1}}, N_{1,2 B_{1}}$ and $P_{1,2 B_{1}}$, and the applied loads $M_{1,2}, N_{1,2}$ and $P_{1,2}$.

$$
\begin{gathered}
R_{A}=-P_{B_{1}} \quad, \quad M_{A}=-M_{B_{1}}-P_{B_{1}} L_{1} \quad, \quad N_{A}=N_{B_{1}} \\
R_{C}=P_{B_{2}}, \quad M_{C}=P_{B_{2}} L_{2}-M_{B_{2}}, \quad N_{C}=N_{B_{2}} \\
M_{B_{1,2}}=M_{1 B_{1,2}}+M_{2 B_{1,2}}+\left(h_{1} N_{2 B_{1,2}}-h_{2} N_{1 B_{1,2}}\right) / 2 \\
N_{B_{1,2}}=N_{1 B_{1,2}}+N_{2 B_{1,2}}, \quad P_{B_{1,2}}=P_{1 B_{1,2}}+P_{2 B_{1,2}} \\
M_{1,2 B_{2}}=M_{1,2 B_{1}}-M_{1,2}-a P_{1,2 B_{1}}+P_{1,2}\left(a-x_{p_{1,2}}\right) \\
N_{1,2 B_{2}}=N_{1,2 B_{1}}-N_{1,2} \quad, \quad P_{1,2 B_{2}}=P_{1,2 B_{1}}-P_{1,2}
\end{gathered}
$$

Eqs. (1) to (7) therefore contain 12 unknown quantities: the six left crack tip forces $M_{1,2 B_{1}}, N_{1,2 B_{1}}$ and $P_{1,2 B_{1}}$, and the deflections, rotations and axial displacements at the left crack tip $w_{1,2 B_{1}}, \psi_{1,2 B_{1}}$ and 
$u_{1,2 B_{1}} .12$ boundary conditions are therefore required to enforce continuity at the crack tip. There is continuity of deflection at the two crack tips.

$$
w_{1 B_{1}}=w_{2 B_{1}}=\left(w_{3}\right)_{x=L_{1}} \quad, \quad\left(w_{1}\right)_{x=a}=\left(w_{2}\right)_{x=a}=\left(w_{4}\right)_{x=0}
$$

There is also continuity of rotation at the two crack tips but this boundary condition requires special consideration. In cases where the shear modulus is finite, but the through-thickness shear effect is still small relative to bending, it is sufficient to use the following approximation:

$$
\psi_{1 B_{1}}=\psi_{2 B_{1}}=\left(\psi_{3}\right)_{x=L_{1}} \quad, \quad\left(\psi_{1}\right)_{x=a}=\left(\psi_{2}\right)_{x=a}=\left(\psi_{4}\right)_{x=0}
$$

In this work, an improved boundary condition is derived and used instead of the approximate Eq. (15). Using a single Timoshenko beam to model the intact region ahead of a crack tip, as in Eqs. (3) to (5), gives constant shear strain through the thickness. In reality, due to the normal and shear stress distributions on the interface ahead of the crack tip, the shear strain is not generally constant through the thickness. Note that the rotations $\psi_{1}$ and $\psi_{2}$ are continuous across the crack tips (although the mid-surface rotations $d w_{1} / d x$ and $d w_{2} / d x$ are not) but they are not equal. One way to represent the mechanics is to model the intact side of each crack tip using two Timoshenko beams with normal and shear stress distributions on the interface and continuous rotations across the crack tip. This would be both complex and incompatible with Eqs. (3) to (5). Instead, the method used in this work, which turns out to be very accurate, is to use a single Timoshenko beam to model the intact side of each crack tip, and account for the presence of the normal and shear stress distributions on the interface with discontinuous rotations $\psi_{1,2}$ across the crack tip. This is justified because the region affected by the crack tip in linear elastic fracture mechanics is small.

Consider the region around a general crack tip, as shown in Figure 1.2. The origin of the $\xi$ coordinate is at the crack tip B and towards the left. The deflection $w$ is upwards and the rotations $d w / d x$ and $\psi$ are defined as positive in the anticlockwise direction. The interface stresses in the figure show only the sign convention rather than any representative distribution. Within the region affected by the crack tip, the through-thickness shearing equations from Timoshenko beam theory are

$$
b h_{1,2} G_{x z} \kappa^{2}\left(d w_{1,2} / d x-\psi_{1,2}\right)=P_{1,2 B} \mp b \int_{0}^{\xi} \sigma_{n} d \xi
$$

The mid-surface rotations $d w_{1} / d x$ and $d w_{2} / d x$ are discontinuous at the crack tip but for a rigid interface, $\left(d w_{1} / d x\right)_{\xi=\delta a}=\left(d w_{2} / d x\right)_{\xi=\delta a}$, which are the midplane rotations of beams 1 and 2 at a very small distance $\delta a$ ahead of the crack tip B. Since the rotations $\psi_{1}$ and $\psi_{2}$ are continuous and $\delta a$ is very small, therefore $\left(\psi_{1,2}\right)_{\xi=\delta a}=\psi_{1,2 B}$. Also, if the intact side of the crack tip is modeled with a 
single Timoshenko beam across the thickness, then $\left(d w_{1,2} / d x\right)_{\xi=\delta a}=(d w / d x)_{B}$. The rotation boundary conditions are therefore

$$
\begin{aligned}
& \psi_{1,2 B_{1}}=\left(d w_{3} / d x\right)_{x=L_{1}}-\left(P_{1,2 B_{1}} \mp F_{n B_{1}}\right) /\left(b h_{1,2} G_{x z} \kappa^{2}\right) \\
& \psi_{1,2 B_{2}}=\left(d w_{4} / d x\right)_{x=0}-\left(P_{1,2 B_{2}} \mp F_{n B_{2}}\right) /\left(b h_{1,2} G_{x z} \kappa^{2}\right)
\end{aligned}
$$

where $F_{n B}=b \int_{0}^{\delta a} \sigma_{n} d \xi$, which is the crack tip opening force. Note that Eqs. (17) and (18) reduce to Eq. (15) for Euler beams, for which $b h G_{x z} \kappa^{2} \rightarrow \infty$. The crack tip opening force $F_{n B}$ is known from the previously established mode partition theory for fracture in isotropic DCBs $(10,12)$. It is dependent on the mode partition and is a function of the crack tip forces. An expression for $F_{n B}$ is given in the following section.

There is also continuity of axial displacement at the two crack tips.

$$
u_{1,2 B_{1}}=\left(u_{3}\right)_{x=L_{1}} \mp h_{2,1}\left(\psi_{3}\right)_{x=L_{1}} / 2 \quad, \quad\left(u_{1,2}\right)_{x=a}=\left(u_{4}\right)_{x=0} \mp h_{2,1}\left(\psi_{4}\right)_{x=0} / 2
$$

The system of 12 equations, given by Eqs. (14) and (17) to (19), can now be solved to give all the unknown quantities in terms of the six independent variables $M_{1,2}, N_{1,2}$ and $P_{1,2}$. The immediate results are not presented here for reasons of conciseness.

\subsection{Energy release rate partition}

ERR can be determined by knowledge of the forces at the crack tip. If all these forces are known, then the ERRs can be found in the same way as for isotropic DCBs $(10,12)$. All of the crack tip forces are known from the solution of the above equations. The mode partition theory for fracture in isotropic DCBs is briefly summarized here. For full details, readers should refer to Refs. (10,12). All expressions are for the left crack tip $B_{1}$. The ' 1 ' subscript is therefore dropped for convenience. It is then simple to find the ERR for the right crack tip $\mathrm{B}_{2}$.

Initially the effect of through-thickness shear is ignored, as in the case of Euler beam theory where $b h G_{x z} \kappa^{2} \rightarrow \infty$. It is accounted for later. The total ERR is

$$
G=\frac{1}{2 b E}\left[\frac{N_{1 B e}^{2} \gamma}{b h_{1}(1+\gamma)}+\frac{M_{1 B}^{2}}{I_{1}}+\frac{M_{2 B}^{2}}{I_{2}}-\frac{1}{I}\left(M_{1 B}+M_{2 B}-\frac{h_{2} N_{1 B e}}{2}\right)^{2}\right]
$$

where $N_{1 B e}$ is the effective axial force, defined as

$$
N_{1 B e}=N_{1 B}-N_{2 B} / \gamma
$$


Since the ERR is a function of three crack tip forces, three pure-mode vectors are required to find the partition. There are eight independent pure-mode vectors to choose from in the Euler case (two puremode-I modes and two pure-mode-II modes from each of the two sets). The order of the variables in the mode vectors is $\varphi=\left\{\begin{array}{lll}M_{1 B} & M_{2 B} & N_{1 B e}\end{array}\right\}^{T}$. It is convenient to select the following three pure modes to make the partition:

$$
\varphi_{\theta_{1}}=\left\{\begin{array}{lll}
1 & \theta_{1} & 0
\end{array}\right\}^{\mathrm{T}} \quad, \quad \varphi_{\beta_{1}}=\left\{\begin{array}{lll}
1 & \beta_{1} & 0
\end{array}\right\}^{\mathrm{T}} \quad, \quad \varphi_{\beta_{2}}=\left\{\begin{array}{lll}
1 & 0 & \beta_{2}
\end{array}\right\}^{\mathrm{T}}
$$

where

$$
\theta_{1}=-\gamma^{2} \quad, \quad \beta_{1}=\gamma^{2}(3+\gamma) /(1+3 \gamma) \quad, \quad \beta_{2}=2(3+\gamma) /\left[h_{1}(\gamma-1)\right]
$$

Mode $\varphi_{\theta_{1}}$ is a pure mode I mode, which has zero relative shearing displacement just behind the crack tip. Modes $\varphi_{\beta_{1}}$ and $\varphi_{\beta_{2}}$ are pure mode II modes, which have zero opening force ahead of the crack tip. Using these modes, the mode partition coefficients are

$$
\left\{\begin{array}{l}
\alpha_{\theta_{1}} \\
\alpha_{\beta_{1}} \\
\alpha_{\beta_{2}}
\end{array}\right\}=\left[\begin{array}{ccc}
1 & 1 & 0 \\
\theta_{1} & \beta_{1} & 0 \\
0 & 0 & 1
\end{array}\right]^{-1}\left\{\begin{array}{l}
M_{1 B} \\
M_{2 B} \\
N_{1 B e}
\end{array}\right\} \quad, \quad\left\{\begin{array}{l}
\alpha_{\theta_{1}} \\
\alpha_{\beta_{1}} \\
\alpha_{\beta_{2}}
\end{array}\right\}=\left[\begin{array}{ccc}
1 & 1 & 1 \\
\theta_{1} & \beta_{1} & 0 \\
0 & 0 & \beta_{2}
\end{array}\right]^{-1}\left\{\begin{array}{l}
M_{1 B} \\
M_{2 B} \\
N_{1 B e}
\end{array}\right\}
$$

for $\gamma=1$ and $\gamma \neq 1$ respectively.

Within the context of Euler beam theory, there is interaction between the $\varphi_{\theta_{1}}$ mode and the $\varphi_{\beta_{i}}$ modes, denoted by $\Delta G_{\theta_{1} \beta_{i}}$. The mode I ERR is

$$
G_{I}=\alpha_{\theta_{1}}^{2} G_{\theta_{1}}+\alpha_{\theta_{1}} \alpha_{\beta_{1}} \Delta G_{\theta_{1} \beta_{1}}+\alpha_{\theta_{1}} \alpha_{\beta_{2}} \Delta G_{\theta_{1} \beta_{2}}
$$

where

$$
G_{\theta_{1}}=24 \gamma /\left[E b^{2} h_{1}^{3}(1+\gamma)\right], \quad \Delta G_{\theta_{1} \beta_{1}}=3(\gamma-1) G_{\theta_{1}} /(1+3 \gamma) \quad, \quad \Delta G_{\theta_{1} \beta_{2}}=\gamma G_{\theta_{1}} /(1+\gamma)
$$

To find the ERR using Timoshenko beam theory, the through-thickness shear effect must be considered. The interaction between the $\varphi_{\theta_{1}}$ mode and the $\varphi_{\beta_{i}}$ modes disappears, that is, $\Delta G_{\theta_{1} \beta_{1,2}}=0$. There are nine independent pure modes within the context of Timoshenko beam theory (there is an extra pure mode I mode due to through-thickness shearing), however the absence of interaction means that four of them from the second set coincide with four of them from the first set, giving five unique pure modes (three pure mode I and two pure mode II). There are also two additional contributions to the mode I ERR $G_{I}$ : the ERR due to shearing, denoted by $G_{P}$; and the 
ERR due to interaction between the $\varphi_{\theta_{1}}$ mode crack tip opening force and the relative opening displacement due to shearing, and vice versa, denoted by $\Delta G_{\theta_{1} P}$. The ERRs are therefore

$$
\begin{gathered}
G=\frac{1}{2 b E}\left[\frac{N_{1 B e}^{2} \gamma}{b h_{1}(1+\gamma)}+\frac{M_{1 B}^{2}}{I_{1}}+\frac{M_{2 B}^{2}}{I_{2}}-\frac{1}{I}\left(M_{1 B}+M_{2 B}-\frac{h_{2} N_{1 B e}}{2}\right)^{2}\right]+G_{P}+\alpha_{\theta_{1}} \Delta G_{\theta_{1} P} \\
G_{I}=\alpha_{\theta_{1}}^{2} G_{\theta_{1}}+G_{P}+\alpha_{\theta_{1}} \Delta G_{\theta_{1} P}
\end{gathered}
$$

where

$$
G_{P}=\frac{1}{2 b h_{1} G_{x z} \kappa^{2}}\left(P_{1 B}^{2}+\frac{P_{2 B}^{2}}{\gamma}-\frac{\left(P_{1 B}+P_{2 B}\right)^{2}}{1+\gamma}\right) \quad, \quad \Delta G_{\theta_{1} P}=\frac{4 \sqrt{3}\left(\gamma P_{1 B}-P_{2 B}\right)}{b^{2} h_{1}^{2}(1+\gamma) \sqrt{E G_{x z} \kappa^{2}}}
$$

The crack tip opening force $F_{n B}$, which is required to solve the governing equations from Timoshenko beam theory in Section 2.1, can now be found. Since the $\varphi_{\beta_{1}}$ and $\varphi_{\beta_{2}}$ modes are characterized by zero normal force ahead of the crack tip, only the $\varphi_{\theta_{1}}$ mode and the opening force due to shearing contribute to the crack tip opening force, giving

$$
F_{n B}=\alpha_{\theta_{1}} F_{n B \theta_{1}}+F_{n B P}
$$

where $\alpha_{\theta_{1}}$ is known in terms of the crack tip forces from Eq. (24) and $F_{n B \theta_{1}}$ is the crack tip opening force in the $\varphi_{\theta_{1}}$ mode and $F_{n B P}$ is the crack tip opening force due to shearing. From Timoshenko beam theory, these quantities are

$$
F_{n B \theta_{1}}=\left(48 G_{x z} \gamma^{2} \kappa^{2} / E h_{1}^{2}(1+\gamma)^{2}\right)^{1 / 2} \quad, \quad F_{n B P}=\left(\gamma P_{1 B}-P_{2 B}\right) /(1+\gamma)
$$

The governing equations in Section 2.1 can now be solved to find the crack tip forces and to obtain the ERR partition.

\subsection{Two sets of orthogonal pure modes}

Since the crack tip forces in Eq. (24) cannot be set independently of the loads applied at $x_{p_{1}}$ and $x_{p_{2}}$, it is clearly not possible to obtain purely mode vectors $\varphi_{\theta_{1}}, \varphi_{\beta_{1}}$, or $\varphi_{\beta_{2}}$ at a given crack tip. In this work these modes are referred to as 'crack tip modes' because they relate crack tip quantities only. Some combinations of these modes however can give pure mode I or II fractures. These modes are now derived for the left crack tip for the special case when $b h G_{x z} \kappa^{2} \rightarrow \infty$ (Euler beam theory) and $\gamma \neq 1 ; P_{1}$ and $P_{2}$ are applied at the same location, i.e. $x_{p_{1}}=x_{p_{2}}=x_{p}$ and $M_{1}=M_{2}=N_{1}=N_{2}=0$. 
These fracture modes are now referred to as ' $F$ modes' and denoted by a subscript ' $F$ ', because they relate the forces $P_{1}$ and $P_{2}$.

Because of Refs. $(10,12)$, there are expected to be two sets of pure F modes, where the first set corresponds to zero relative shearing displacement just behind the crack tip (pure mode I) and zero opening force ahead of the crack tip (pure mode II); and where the second set corresponds to zero relative opening displacement just behind the crack tip (pure mode II) and zero crack tip shearing force (pure mode I).

Mathematically, the relative shearing displacement at an infinitely small distance $\delta a$ behind the crack tip, $\left(D_{s h}\right)_{x=\delta a}$ is expressed as

$$
\left(D_{s h}\right)_{x=\delta a}=\left(\bar{u}_{1}\right)_{x=\delta a}-\left(\bar{u}_{2}\right)_{x=\delta a}=\left(u_{1}\right)_{x=\delta a}+h_{1}\left(\psi_{1}\right)_{x=\delta a} / 2-\left(u_{2}\right)_{x=\delta a}+h_{2}\left(\psi_{2}\right)_{x=\delta a} / 2
$$

where $\bar{u}$ represents the axial displacement at the interface. For the pure-mode-I mode from the first set, the relative shearing displacement at $x=\delta a$ is zero, that is $\left(D_{s h}\right)_{x=\delta a}=0$. Making the necessary substitutions and taking the limit as $\delta a \rightarrow 0$ gives

$$
P_{2} / P_{1}=\theta_{F}=-\left[\gamma^{2}\left(C_{1} \gamma^{2}-C_{2} \gamma+C_{3}\right)\right] /\left(C_{3} \gamma^{2}-C_{2} \gamma+C_{1}\right) \quad, \quad \varphi_{\theta_{F}}=\left\{\begin{array}{ll}
1 & \theta_{F}
\end{array}\right\}^{T}
$$

where

$$
\begin{gathered}
C_{1}=2 x_{p}\left(a-x_{p}\right)^{2}\left(a+L_{1}+L_{2}\right)^{3} \quad, \quad C_{2}=2 x_{p}\left(a-x_{p}\right)^{2}\left(C_{7}+3 a^{2}\left(L_{1}+L_{2}\right)-2 a^{3}\right) \\
C_{3}=2 a^{3} x_{p}^{3}-a x_{p}^{2}\left(C_{7}+6 a^{2} L_{2}+4 a^{3}\right)+2 a^{2} x_{p}\left(C_{7}-3 a L_{1} L_{2}+3 a^{2} L_{2}+a^{3}\right)-a C_{8} \\
C_{7}=\left(L_{1}+L_{2}\right)^{3}+3 a\left(L_{1}+L_{2}\right)^{2} \quad, \quad C_{8}=a^{2} L_{1}^{2}\left(L_{1}+3 L_{2}+3 a\right)
\end{gathered}
$$

and $\varphi_{F}$ represents mode vector format $\left\{\begin{array}{ll}P_{1} & P_{2}\end{array}\right\}^{T}$. The orthogonal condition to the zero relative shearing displacement condition is zero opening force ahead of the crack tip. The mode corresponding to this condition could be derived by applying orthogonality through Eq. (20), however it is more convenient in this instance to simply enforce $F_{n B}=0$. Noting that $F_{n B \theta_{1}}$ is infinite in Euler beam theory and that therefore the finite $F_{n B P}$ in Eq. (30) is negligible, the requirement for $F_{n B}=0$ is simply $\alpha_{\theta_{1}}=0$, which from Eq. (24) gives

$$
P_{2} / P_{1}=\beta_{F}=\gamma^{2}\left(C_{1} \gamma^{3}+C_{4} \gamma^{2}+C_{5} \gamma+C_{6}\right) /\left(C_{6} \gamma^{3}+C_{5} \gamma^{2}+C_{4} \gamma+C_{1}\right), \varphi_{\beta_{F}}=\left\{\begin{array}{ll}
1 & \beta_{F}
\end{array}\right\}^{T}
$$

where

$$
C_{4}=2 x_{p}\left(a-x_{p}\right)^{2}\left(2 C_{7}+6 a^{2}\left(L_{1}+L_{2}\right)+5 a^{3}\right)
$$




$$
\begin{gathered}
C_{5}=x_{p}^{3}\left(2 C_{7}+6 a^{2}\left(L_{1}+L_{2}\right)+14 a^{3}\right)-a x_{p}^{2}\left(C_{7}+12 a^{2} L_{1}-6 a^{2} L_{2}+28 a^{3}\right) \\
-2 a^{2} x_{p}\left(2 C_{7}-3 a^{2} L_{1}+6 a^{2} L_{2}-7 a^{3}-9 a L_{1} L_{2}\right)+3 a C_{8} \\
C_{6}=6 a^{3} x_{p}^{3}-3 a x_{p}^{2}\left(C_{7}+6 a^{2} L_{2}+4 a^{3}\right)+6 a^{2} x_{p}\left(C_{7}-3 a L_{1} L_{2}+3 a^{2} L_{2}+a^{3}\right)-3 a C_{8}
\end{gathered}
$$

Now considering the second set of pure modes, the pure mode I mode is given by zero shear force at the crack tip. The shear force at the crack tip is

$$
F_{s B}=\alpha_{\theta_{1}} F_{s B \theta_{1}}+\alpha_{\beta_{1}} F_{s B \beta_{1}}+\alpha_{\beta_{2}} F_{s B \beta_{2}}
$$

The mode partition coefficients are known from Eq. (24) and the crack tip shear forces relating to each mode vector are known from Refs. $(10,12)$.

$$
F_{s B \theta_{1}}=6 \gamma(1-\gamma) /\left[h_{1}(1+\gamma)^{2}\right] \quad, \quad F_{s B \beta_{1}}=6 \gamma /\left[h_{1}(1+3 \gamma)\right] \quad, \quad F_{s B \beta_{2}}=2 \gamma^{2} /\left[h_{1}\left(\gamma^{2}-1\right)\right]
$$

Making these substitutions and setting $F_{s B}=0$ gives

$$
P_{2} / P_{1}=\theta_{F}^{\prime}=-1 \quad, \quad \varphi_{\theta_{F}^{\prime}}=\left\{\begin{array}{ll}
1 & \theta_{F}^{\prime}
\end{array}\right\}^{T}
$$

Finally, for the pure mode II mode from the second set, the relative opening displacement at $x=\delta a$ is zero $\left(D_{o p}\right)_{x=\delta a}=0$.

$$
\left(D_{o p}\right)_{x=\delta a}=\left(w_{1}\right)_{x=\delta a}-\left(w_{2}\right)_{x=\delta a}
$$

Making the necessary substitutions and taking the limit as $\delta a \rightarrow 0$ gives

$$
P_{2} / P_{1}=\beta_{F}^{\prime}=\gamma^{3} \quad, \quad \varphi_{\beta_{F}^{\prime}}=\left\{\begin{array}{ll}
1 & \beta_{F}^{\prime}
\end{array}\right\}^{T}
$$

That $\theta_{F}^{\prime}$ and $\beta_{F}^{\prime}$, relating $P_{2}$ to $P_{1}$, are the same as $\theta_{1}^{\prime}$ and $\beta_{1}^{\prime}$, which relate $M_{2 B}$ to $M_{1 B}(10,12)$, should be no surprise. The axial forces $N_{1 B}$ and $N_{2 B}$, induced at the crack tip by $P_{1}$ and $P_{2}$, clearly have no effect on the opening displacement. Therefore the condition $\left(D_{o p}\right)_{x=\delta a}=0$ is unaffected by $N_{1 B}$ and $N_{2 B}$, and $\beta_{F}^{\prime}=\beta_{1}^{\prime}$. Also, if $P_{1}$ and $P_{2}$ are equal and opposite $P_{2} / P_{1}=-1$, then regardless of how beams 1 and 2 deflect, beams 3 and 4 do not deflect and the crack tip rotations are zero.

Therefore $N_{1 B}$ and $N_{2 B}$ are both zero and the two crack tip bending moments are in the ratio of $\theta_{1}^{\prime}$. If $P_{1}$ and $P_{2}$ are applied in different locations then this would not be observed because each load would have a different moment arm around the crack tip.

If the above procedure is repeated for Timoshenko beams, it is found that the $\varphi_{\theta_{F}^{\prime}}$ mode changes to coincide with the $\varphi_{\theta_{F}}$ mode, which is different to that obtained from Euler beam theory due to the static indeterminacy of clamped-clamped beams (the relative shearing displacement is otherwise not 
affected by through-thickness shear). Similarly the $\varphi_{\beta_{F}^{\prime}}$ mode coincides with the $\varphi_{\beta_{F}}$ mode, which is also different to that obtained from Euler beam theory. For cases when the through-thickness shear effect is not excessively large, Eqs. (33) and (37) are good approximations. The expressions for the Timoshenko ' $F$ modes' are not as simple as those for the Euler ' $F$ modes', so are not presented here in general form. They are however easily derived for specific cases with numerical—rather than algebraic—quantities.

\subsection{Contacting fractures}

For some values of $P_{1}$ and $P_{2}$, the beams either side of the fracture will come into contact. This raises two questions: where is the point of first contact, and what happens after contact?

To find the point of first contact $x_{c}$ using Euler beam theory, two conditions must be satisfied: first, the relative opening displacement at this point must be zero; second, the relative opening displacement must be a minimum at $x=x_{c}$, which implies that it is the point of first contact.

$$
\left(D_{o p}\right)_{x=x_{c}}=0 \quad, \quad\left(\partial D_{o p} / \partial x\right)_{x=x_{c}}=0
$$

Solving Eqs. (46) simultaneously for $P_{2} / P_{1}$ and $x_{c}$, and ignoring the obvious and unavailing solutions for the crack tips, gives

$$
P_{2} / P_{1}=\gamma^{3}=\beta_{F}^{\prime}
$$

for all values of $x$. This implies simultaneous contact everywhere along the fracture for this value of $P_{2} / P_{1}$.

If $P_{2} / P_{1}$ is increased beyond $\beta_{F}^{\prime}$, the contact can either be at a point or distributed. Obviously the solution must not allow interpenetration between the upper and lower beams anywhere. In addition, since linear elastic mechanics is being used, there can only be one valid solution. Therefore point contact at $x=x_{p}$, which is a reasonable assumption, will be considered and shown to satisfy the requirements, thus demonstrating that it is the correct solution.

Say that two loads $P_{1 c}$ and $P_{2 c}$ are applied to the beam at $x=x_{p}$ and that they cause point contact at this same location. Call the point contact force $P_{c}$. It acts to prevent non-physical intersection. The net shear loads $P_{1}$ and $P_{2}$ acting on the beams are therefore

$$
P_{1}=P_{1 c}+P_{c} \quad, \quad P_{2}=P_{2 c}-P_{c}
$$


Note that $P_{1}$ and $P_{2}$ in Eqs. (48) are the same quantities that appear in all the equations thus far. The final condition that must be satisfied is

$$
\left(D_{o p}\right)_{x=x_{p}}=0
$$

Solving Eqs. (48) and (49) for $P_{1}, P_{2}$ and $P_{c}$ gives

$$
P_{1}=\left(P_{1 c}+P_{2 c}\right) /\left(1+\gamma^{3}\right) \quad, \quad P_{2}=\gamma^{3}\left(P_{1 c}+P_{2 c}\right) /\left(1+\gamma^{3}\right) \quad, \quad P_{c}=\left(P_{2 c}-\gamma^{3} P_{1 c}\right) /\left(1+\gamma^{3}\right)
$$

Substituting Eqs. (50) into $D_{o p}=w_{1}-w_{2}$ reveals that $D_{o p}=0$ for all values of $x$. Therefore the requirements for physical contact behavior are satisfied by this solution and the ERR partition is then found in the usual way.

Using Timoshenko beam theory, at $P_{2} / P_{1}=\beta_{F}=\beta_{F}^{\prime}$ there is both zero opening force ahead of the crack tip and zero relative opening displacement just behind. Therefore crack tip running contact occurs at $P_{2} / P_{1}=\beta_{F}$ and a pure mode II fracture is obtained. Since there is running contact, if the loading ratio $P_{2} / P_{1}$ is increased further then the crack tip remains closed as the contacting region grows.

\section{Simply supported isotropic beams}

The theory presented in Section 2 is easily modified for simply supported isotropic beams. In this section, the modified theory is briefly summarized. For this new case, Eqs. (51) to (53) replace Eqs. (3) to (5) and (7).

$$
\begin{gathered}
\psi_{3}=\frac{1}{E I_{3}}\left(\frac{R_{A} x^{2}}{2}-M_{A} x\right)+\psi_{A} \quad, \quad w_{3}=\frac{1}{E I_{3}}\left(\frac{R_{A} x^{3}}{6}-\frac{M_{A} x^{2}}{2}\right)+\psi_{A} x+\frac{P_{B_{1}} x}{b h G_{x z} \kappa^{2}} \\
\psi_{4}=\frac{1}{E I_{4}}\left(M_{B_{2}} x-\frac{P_{B_{2}} x^{2}}{6}\right)+\psi_{B_{2}} \\
w_{4}=\frac{1}{E I_{4}}\left(\frac{M_{B_{2}} x^{2}}{2}-\frac{M_{B_{2}} L_{2}{ }^{2}}{2}-\frac{P_{B_{2}} x^{3}}{6}+\frac{P_{B_{2}} L_{2}{ }^{3}}{6}\right)+\psi_{B_{2}}\left(x-L_{2}\right)+\frac{P_{B_{2}}\left(x-L_{2}\right)}{b h G_{x z} \kappa^{2}}
\end{gathered}
$$

Since zero rotation is no longer enforced at the supports, two additional boundary conditions are required at these locations. For simple supports these are

$$
M_{A}=0=-P_{B_{1}} L_{1}-M_{B_{1}} \quad, \quad M_{C}=0=P_{B_{2}} L_{2}-M_{B_{2}}
$$

This new system of equations is now easily solved for the left crack tip for the special case when $b h G_{x z} \kappa^{2} \rightarrow \infty$ (Euler beam theory). All the unknown quantities are then known in terms of the 
independent variables $P_{1}$ and $P_{2}$. Again, the immediate results are not presented here for reasons of conciseness. If the analysis is carried out for when $\gamma \neq 1, P_{1}$ and $P_{2}$ are applied at the same location, i.e. $x_{p_{1}}=x_{p_{2}}=x_{p}$ and $M_{1}=M_{2}=N_{1}=N_{2}=0$, the four pure mode relationships for both the left and right crack tips are

$$
\begin{gathered}
P_{2} / P_{1}=\theta_{F}=\gamma^{2}\left(C_{1} \gamma^{2}-C_{1} \gamma-C_{2}\right) /\left(C_{2} \gamma^{2}+C_{1} \gamma-C_{1}\right) \\
P_{2} / P_{1}=\beta_{F}=\gamma^{2}\left(C_{1} \gamma^{3}+2 C_{1} \gamma^{2}+C_{3} \gamma+C_{4}\right) /\left(C_{4} \gamma^{3}+C_{3} \gamma^{2}+2 C_{1} \gamma+C_{1}\right) \\
P_{2} / P_{1}=\theta_{F}^{\prime}=-1 \quad, \quad P_{2} / P_{1}=\beta_{F}^{\prime}=\gamma^{3}
\end{gathered}
$$

where

$$
\begin{gathered}
C_{1}=2 x_{p}\left(a-x_{p}\right)^{2}\left(a+L_{1}+L_{2}\right) \quad, \quad C_{2}=C_{5} x_{p}^{2}+a x_{p}\left(a^{2}-2 C_{5}\right)+a^{3} L_{1} \\
C_{3}=2 x_{p}^{3}\left(a+L_{1}+L_{2}\right)-C_{5} x_{p}^{2}+a x_{p}\left(3 a^{2}-4 C_{5}\right)+3 a^{3} L_{1} \\
C_{4}=-3 C_{5} x_{p}^{2}+a x_{p}\left(6 C_{5}-3 a^{2}\right)-3 a^{3} L_{1} \quad, \quad C_{5}=a\left(a+L_{1}+L_{2}\right)
\end{gathered}
$$

The contact behavior is found to be identical to the clamped-clamped case, i.e. contact at $P_{2} / P_{1}=\gamma^{3}$ for all values of $x$ between beams 1 and 2 and point contact at $x=x_{p}$ afterwards.

If the above procedure is repeated for Timoshenko beams then as before, it is found that the $\varphi_{\theta_{F}^{\prime}}$ mode coincides with the $\varphi_{\theta_{F}}$ mode; the $\varphi_{\beta_{F}^{\prime}}$ mode coincides with the $\varphi_{\beta_{F}}$ mode; and the $\varphi_{\theta_{F}}$ and $\varphi_{\beta_{F}}$ modes are different to those obtained from Euler beam theory. However for cases when the through-thickness shear effect is not excessively large, Eqs. (55) and (56) are good approximations. The expressions for the Timoshenko ' $\mathrm{F}$ modes' are not as simple as those for the Euler ' $\mathrm{F}$ modes', so are not presented here in general form. They are however easily derived for specific cases with numerical quantities.

\section{Clamped-clamped laminated composite beams}

\subsection{Governing equations}

A general clamped-clamped laminated composite beam with a delamination now receives the same analysis. Contact between the upper and lower sub-laminates is not treated initially. The extensional, coupling, bending and shearing stiffness are denoted by $A, B, D$ and $H$ respectively. Note that these quantities take different values under the plane-strain assumption from those from under the plane-stress assumption however they make no difference to the following development. Subscripts 1 and 2 are used to indicate the upper and lower sub-laminates respectively. No subscript is used for the 
intact part of the laminate. $A_{1}$ is therefore the extensional stiffness of the upper sub-laminate and $A$ is the extensional stiffness of the intact laminate, etc. With reference to Figure 1.1 and using the constitutive relation from classical laminate theory,

$$
\begin{gathered}
\left\{\begin{array}{c}
N_{1,2}(x) / b \\
-M_{1,2}(x) / b \\
P_{1,2}(x) / b
\end{array}\right\}=\left[\begin{array}{ccc}
A_{1,2} & B_{1,2} & 0 \\
B_{1,2} & D_{1,2} & 0 \\
0 & 0 & H_{1,2}
\end{array}\right]\left\{\begin{array}{c}
d u_{1,2} / d x \\
-d \psi_{1,2} / d x \\
d w_{1,2} / d x-\psi_{1,2}
\end{array}\right\} \\
\left\{\begin{array}{c}
N_{3,4}(x) / b \\
-M_{3,4}(x) / b \\
P_{3,4}(x) / b
\end{array}\right\}=\left[\begin{array}{ccc}
A & B & 0 \\
B & D & 0 \\
0 & 0 & H
\end{array}\right]\left\{\begin{array}{c}
d u_{3,4} / d x \\
-d \psi_{3,4} / d x \\
d w_{3,4} / d x-\psi_{3,4}
\end{array}\right\}
\end{gathered}
$$

where

$$
\begin{gathered}
N_{1,2}(x)=N_{1,2 B_{1}}-N_{1,2}\left\langle x-x_{p_{1,2}}\right\rangle^{0}, \quad P_{1,2}(x)=P_{1,2 B_{1}}-P_{1,2}\left\langle x-x_{p_{1,2}}\right\rangle^{0} \\
M_{1,2}(x)=M_{1,2 B_{1}}-P_{1,2 B_{1}} x-M_{1,2}\left\langle x-x_{p_{1,2}}\right\rangle^{0}+P_{1,2}\left\langle x-x_{p_{1,2}}\right\rangle \\
N_{3,4}(x)=N_{B_{1,2}}, \quad P_{3,4}(x)=P_{B_{1,2}} \\
M_{3}(x)=M_{B_{1}}-P_{B_{1}}\left(x-L_{1}\right) \quad, \quad M_{4}(x)=M_{B_{2}}-P_{B_{2}} x
\end{gathered}
$$

As shown in Figure 1.1a, the origin of $x$ in these equations depends on the beam in question: it is at location $\mathrm{B}_{1}$ and to the right for beams 1 and 2; for beams 3 and 4 it is at the respective left-hand sides and to the right; Positive deflection, $w$ is always upwards; the rotations $d w / d x$ and $\psi$ are positive in the anticlockwise direction. From Eqs. (61) to (66), the following are easily derived:

$$
\begin{gathered}
\psi_{1,2}=\frac{B_{1,2}\left(N_{1,2 B_{1}} x-N_{1,2}\left\langle x-x_{p_{1,2}}\right\rangle\right)}{b\left(A_{1,2} D_{1,2}-B_{1,2}^{2}\right)}+\psi_{1,2 B_{1}} \\
+\frac{A_{1,2}\left(2 M_{1,2 B_{1}} x-2 M_{1,2}\left\langle x-x_{p_{1,2}}\right\rangle+P_{1,2}\left\langle x-x_{p_{1,2}}\right\rangle^{2}-P_{1,2 B_{1}} x^{2}\right)}{2 b\left(A_{1,2} D_{1,2}-B_{1,2}^{2}\right)} \\
w_{1,2}=\frac{B_{1,2}\left(N_{1,2 B_{1}} x^{2}-N_{1,2}\left\langle x-x_{p_{1,2}}\right\rangle^{2}\right)}{2 b\left(A_{1,2} D_{1,2}-B_{1,2}^{2}\right)}+\psi_{1,2 B_{1}} x+w_{1,2 B_{1}}+\frac{P_{1,2 B_{1}} x-P_{1,2}\left\langle x-x_{p_{1,2}}\right\rangle}{b H_{1,2}} \\
+\frac{A_{1,2}\left(3 M_{1,2 B_{1}} x^{2}-3 M_{1,2}\left\langle x-x_{p_{1,2}}\right\rangle^{2}+P_{1,2}\left\langle x-x_{p_{1,2}}\right\rangle^{3}-P_{1,2 B_{1}} x^{3}\right)}{6 b\left(A_{1,2} D_{1,2}-B_{1,2}^{2}\right)} \\
\psi_{3}=\frac{2 B N_{B_{1}} x+2 A M_{B_{1}} x+2 A L_{1} P_{B_{1}} x-A P_{B_{1}} x^{2}}{2 b\left(A D-B^{2}\right)}
\end{gathered}
$$




$$
\begin{gathered}
w_{3}=\frac{3 B N_{B_{1}} x^{2}+3 A M_{B_{1}} x^{2}+3 A L_{1} P_{B_{1}} x^{2}-A P_{B_{1}} x^{3}}{6 b\left(A D-B^{2}\right)}+\frac{P_{B_{1}} x}{b H} \\
w_{4}=\frac{\left(2 B N_{B_{2}}+2 A M_{B_{2}}\right)\left(x-L_{2}\right)-A P_{B_{2}}\left(x^{2}-L_{2}^{2}\right)}{2 b\left(A D-B^{2}\right)} \\
w_{4}=\frac{\left(3 B N_{B_{2}}+3 A M_{B_{2}}\right)\left(x^{2}-2 L_{2} x+L_{2}^{2}\right)-A P_{B_{2}}\left(x^{3}-3 L_{2}^{2} x+2 L_{2}^{2}\right)}{6 b\left(A D-B^{2}\right)}+\frac{P_{B_{2}}\left(x-L_{2}\right)}{b H} \\
u_{1,2}=\frac{D_{1,2}\left(N_{1,2 B_{1}} x-N_{1,2}\left\langle x-x_{p_{1,2}}\right\rangle\right)}{b\left(A_{1,2} D_{1,2}-B_{1,2}^{2}\right)}+u_{1,2 B_{1}} \\
+\frac{B_{1,2}\left(2 M_{1,2 B_{1}} x-2 M_{1,2}\left\langle x-x_{p_{1,2}}\right\rangle+P_{1,2}\left\langle x-x_{p_{1,2}}\right\rangle^{2}-P_{1,2 B_{1}} x^{2}\right)}{2 b\left(A_{1,2} D_{1,2}-B_{1,2}^{2}\right)} \\
u_{4}=\frac{2 D N_{B_{2}}\left(x-L_{2}\right)+2 B M_{B_{2}}\left(x-L_{2}\right)-B P_{B_{2}}\left(x^{2}-L_{2}^{2}\right)}{2 b\left(A D-B^{2}\right)} \\
u_{3}=\frac{2 D N_{B_{1}} x+2 B M_{B_{1}} x+2 B L_{1} P_{B_{1}} x-B P_{B_{1}} x^{2}}{2 b\left(A D-B^{2}\right)}
\end{gathered}
$$

As before, there are 12 unknown quantities: the six left crack tip forces $M_{1,2 B_{1}}, N_{1,2 B_{1}}$ and $P_{1,2 B_{1}}$, and the deflections, rotations and axial displacements at the left crack tip $w_{1,2 B_{1}}, \psi_{1,2 B_{1}}$ and $u_{1,2 B_{1}}$. Eqs. (14) and (19) are still applicable. The continuity of rotation at the two crack tips is treated in the same way as in Section 2.1 and the following boundary conditions are obtained:

$$
\begin{aligned}
& \psi_{1,2 B_{1}}=\left(d w_{3} / d x\right)_{x=L_{1}}-\left(P_{1,2 B_{1}} \mp F_{n B_{1}}\right) /\left(b H_{1,2}\right) \\
& \psi_{1,2 B_{2}}=\left(d w_{4} / d x\right)_{x=0}-\left(P_{1,2 B_{2}} \mp F_{n B_{2}}\right) /\left(b H_{1,2}\right)
\end{aligned}
$$

Note that Eqs. (76) and (77) reduce to Eq. (15) for Euler beams, for which $b H \rightarrow \infty$. The crack tip opening force $F_{n B}$ is known from the previously established mode partition theory for onedimensional fracture in laminated composite DCBs $(10,13)$. It is dependent on the mode partition and is a function of the crack tip forces. An expression for $F_{n B}$ is given in the following section.

The algebraic solution for the general case is extensive. The solution for the much simpler symmetric case with Euler beams is instead given for reasons of practicality. From symmetry we have

$$
L_{1}=L_{2}=L \quad, \quad x_{p_{1}}=x_{p_{2}}=a / 2 \quad, \quad M_{1}=M_{2}=N_{1}=N_{2}=0
$$

Symmetry provides two additional boundary conditions, which simplify the calculations. These are zero axial displacement and zero rotation at the mid-span. 


$$
\left(u_{1}\right)_{x=a / 2}=\left(u_{2}\right)_{x=a / 2} \quad, \quad\left(\psi_{1}\right)_{x=a / 2}=\left(\psi_{2}\right)_{x=a / 2}
$$

The resulting crack tip forces are

$$
\begin{gathered}
M_{1,2 B_{1}}=C_{1} P_{1,2} \mid 2 a A_{1} A_{2} L^{2}\left(h_{1}+h_{2}\right)^{2}-8 A_{1,2} L^{2}\left(2 D_{1,2} L-a D_{2,1} \pm a h_{1} B_{2,1} \pm a h_{2} B_{2,1}\right) \\
+4 A_{2,1} L^{2}\left(-4 D_{1,2} L+2 a D_{2,1} \pm a h_{1} B_{1,2} \pm a h_{2} B_{1,2}-2 h_{1} B_{1,2} L-2 h_{2} B_{1,2} L\right) \\
+a A L\left(a h_{1}^{2} A_{2}+a h_{2}^{2} A_{1} \mp 4 a h_{1,2} B_{2,1} \pm 2 a h_{2,1} B_{1,2} \mp 4 h_{2,1} B_{1,2} L-8 D_{1,2} L+4 a D_{2,1}\right) \\
+4 a B L\left(-a B_{1,2}+2 B_{1,2} L-2 a B_{2,1}+a h_{1} A_{2}-a h_{2} A_{1}\right)+4 a^{2} D L\left(A_{1}+A_{2}\right) \\
\left.+8 L^{2}\left(2 B_{1,2}^{2} L+2 B_{1} B_{2} L-a B_{2,1}^{2}-a B_{1} B_{2}\right)-2 a^{3} B^{2}+2 a^{3} A D\right] \\
-2 C_{1} L P_{2,1}(a+2 L)\left[-4 B_{1,2} L\left(B_{1}+B_{2}\right)+4 D_{1,2} L\left(A_{1}+A_{2}\right)\right. \\
\left.+a A\left(2 D_{1,2} \pm h_{2,1} B_{1,2}\right)-2 a B B_{1,2} \pm 2 A_{2,1} B_{1,2} L\left(h_{1}+h_{2}\right)\right] \\
N_{1,2 B_{1}}= \pm 2 C_{1} L(a+2 L)\left(P_{1}+P_{2}\right)\left[2 A_{1} A_{2} L\left(h_{1}+h_{2}\right)+a h_{2,1} A A_{1,2}\right. \\
\left.+4 L\left(A_{2} B_{1}-A_{1} B_{2}\right) \pm 2 a\left(A B_{1,2}-A_{1,2} B\right)\right]
\end{gathered}
$$

where

$$
\begin{aligned}
C_{1} & =\left[64 A_{1} L^{2}\left(D_{1}+D_{2}-h_{1} B_{2}-h_{2} B_{2}\right)+64 A_{2} L^{2}\left(D_{1}+D_{2}+h_{1} B_{1}+h_{2} B_{1}\right)\right. \\
& +32 a L\left(A_{1} D+A D_{1}+A_{2} D+A D_{2}-h_{2} A_{1} B+h_{1} A_{2} B+h_{2} A B_{1}-h_{1} A B_{2}\right) \\
& +16 A_{1} A_{2} L^{2}\left(h_{1}+h_{2}\right)^{2}+8 a A L\left(h_{2}^{2} A_{1}+h_{1}^{2} A_{2}\right)-64 L^{2}\left(B_{1}+B_{2}\right)^{2} \\
& \left.-64 a B L\left(B_{1}+B_{2}\right)+16 a^{2}\left(A D-B^{2}\right)\right]^{-1}
\end{aligned}
$$

\subsection{Energy release rate partition}

All of the crack tip forces are known from the solution of the above equations. The ERRs can therefore be found. The mode partition theory for one-dimensional fracture in laminated composite DCBs is briefly summarized here. For full details, readers should refer to works $(10,13)$. All expressions are for the left crack tip $B_{1}$. The ' 1 ' subscript is therefore dropped for convenience. It is then simple to find the ERR for the right crack tip $B_{2}$.

Initially the effect of through-thickness shear is ignored, as in the case of Euler beam theory where $b H \rightarrow \infty$. It is accounted for later. The total ERR is

$$
G=\frac{1}{2 b^{2}}\left(\frac{M_{1 B}^{2}}{D_{1}^{*}}+\frac{M_{2 B}^{2}}{D_{2}^{*}}-\frac{M_{B}^{2}}{D^{*}}+\frac{N_{1 B}^{2}}{A_{1}^{*}}+\frac{N_{2 B}^{2}}{A_{2}^{*}}-\frac{N_{B}^{2}}{A^{*}}-\frac{2 B_{1} M_{1 B} N_{1 B}}{B_{1}^{*}}-\frac{2 B_{2} M_{2 B} N_{2 B}}{B_{2}^{*}}+\frac{2 B M_{B} N_{B}}{B^{*}}\right)
$$

where

$$
A_{i}^{*}=A_{i}-B_{i}^{2} / D_{i} \quad, \quad B_{i}^{*}=B_{i}^{2}-A_{i} D_{i} \quad, \quad D_{i}^{*}=D_{i}-B_{i}^{2} / A_{i}
$$


The range of subscript $i$ is 1 to 2 , which again refers to the upper and lower sub-laminates respectively. For the intact part of the laminate, no subscript is used. Other terms in Eq. (83) are

$$
N_{B}=N_{1 B}+N_{2 B} \quad, \quad M_{B}=M_{1 B}+M_{2 B}+\frac{1}{2}\left(h_{1} N_{2 B}-h_{2} N_{1 B}\right)
$$

Since the ERR is a function of four crack tip forces, four pure modes are required to find the partition. There are 12 pure modes to choose from in the Euler case (six pure mode I and six pure mode II). The order of the variables in the mode vectors is $\varphi=\left\{\begin{array}{llll}M_{1 B} & M_{2 B} & N_{1 B} & N_{2 B}\end{array}\right\}^{T}$. It is convenient to select the following four modes to make the partition:

$$
\begin{aligned}
\varphi_{\theta_{1}} & =\left\{\begin{array}{llll}
1 & \theta_{1} & 0 & 0
\end{array}\right\}^{\mathrm{T}}, \varphi_{\beta_{1}}=\left\{\begin{array}{lllll}
1 & \beta_{1} & 0 & 0
\end{array}\right\}^{T} \\
\varphi_{\beta_{2}} & =\left\{\begin{array}{lllll}
1 & 0 & \beta_{2} & 0
\end{array}\right\}^{T}, \quad \varphi_{\beta_{3}}=\left\{\begin{array}{lllll}
1 & 0 & 0 & \beta_{3}
\end{array}\right\}^{T}
\end{aligned}
$$

where

$$
\begin{gathered}
\theta_{1}=\left(B_{2}^{2}-A_{2} D_{2}\right)\left(B_{1}+h_{1} A_{1} / 2\right) /\left[\left(B_{1}^{2}-A_{1} D_{1}\right)\left(B_{2}-h_{2} A_{2} / 2\right)\right] \\
\beta_{1}=-D_{2}^{*}\left(D_{1}^{*}+D_{1}^{*} \theta_{1}-D^{*}\right) /\left[D_{1}^{*}\left(D_{2}^{*}+D_{2}^{*} \theta_{1}-D^{*} \theta_{1}\right)\right] \\
\beta_{2}=\frac{\theta_{2}\left[h_{2} /\left(2 D^{*}\right)-B_{1} / B_{1}^{*}+B / B^{*}\right]+1 / D_{1}^{*}-1 / D^{*}}{\theta_{2}\left[B h_{2} / B^{*}-1 / A_{1}^{*}+1 / A^{*}+h_{2}^{2} /\left(4 D^{*}\right)\right]-h_{2} /\left(2 D^{*}\right)+B_{1} / B_{1}^{*}-B / B^{*}} \\
\beta_{3}=\frac{\theta_{3}\left[h_{1} /\left(2 D^{*}\right)-B / B^{*}\right]-1 / D_{1}^{*}+1 / D^{*}}{\theta_{3}\left[B h_{1} / B^{*}+1 / A_{2}^{*}-1 / A^{*}-h_{1}^{2} /\left(4 D^{*}\right)\right]-h_{1} /\left(2 D^{*}\right)+B / B^{*}} \\
\left\{\begin{array}{l}
\alpha_{\theta_{1}} \\
\alpha_{\beta_{1}} \\
\alpha_{\beta_{2}} \\
\alpha_{\beta_{3}}
\end{array}\right\}=\left\{\begin{array}{c}
\left(M_{2 B} \beta_{2}+N_{1 B} \beta_{1}-M_{1 B} \beta_{1} \beta_{2}\right) / \beta_{2}\left(\theta_{1}-\beta_{1}\right)+N_{2 B} \beta_{1} /\left[\beta_{3}\left(\theta_{1}-\beta_{1}\right)\right] \\
\left(M_{1 B} \theta_{1} \beta_{2}-M_{2 B} \beta_{2}-N_{1 B} \theta_{1}\right) /\left[\beta_{2}\left(\theta_{1}-\beta_{1}\right)\right]-N_{2 B} \theta_{1} /\left[\beta_{3}\left(\theta_{1}-\beta_{1}\right)\right] \\
N_{1 B} / \beta_{2} \\
N_{2 B} / \beta_{3}
\end{array}\right\}
\end{gathered}
$$

Within the context of Euler beam theory, which has interaction between the $\varphi_{\theta_{1}}$ mode and the $\varphi_{\beta_{i}}$ modes, the mode I ERR is

$$
G_{I}=\alpha_{\theta_{1}}^{2} G_{\theta_{1}}+\alpha_{\theta_{1}} \alpha_{\beta_{1}} \Delta G_{\theta_{1} \beta_{1}}+\alpha_{\theta_{1}} \alpha_{\beta_{2}} \Delta G_{\theta_{1} \beta_{2}}+\alpha_{\theta_{1}} \alpha_{\beta_{3}} \Delta G_{\theta_{1} \beta_{3}}
$$

where

$$
\begin{gathered}
G_{\theta_{1}}=\frac{1}{2 b^{2}}\left[\frac{1}{D_{1}^{*}}+\frac{\theta_{1}^{2}}{D_{2}^{*}}-\frac{\left(1+\theta_{1}\right)^{2}}{D^{*}}\right], \quad \Delta G_{\theta_{1} \beta_{1}}=\frac{F_{n B \theta_{1}} \delta a}{4 b^{2}}\left(\frac{1}{D_{1}^{*}}-\frac{\beta_{1}}{D_{2}^{*}}\right) \\
\Delta G_{\theta_{1} \beta_{2}}=\frac{F_{n B \theta_{1}} \delta a}{4 b^{2}}\left(\frac{1}{D_{1}^{*}}-\frac{B_{1} \beta_{2}}{B_{1}^{*}}\right) \quad, \quad \Delta G_{\theta_{1} \beta_{3}}=\frac{F_{n B \theta_{1}} \delta a}{4 b^{2}}\left(\frac{1}{D_{1}^{*}}+\frac{B_{2} \beta_{3}}{B_{2}^{*}}\right)
\end{gathered}
$$




$$
F_{n B \theta_{1}} \delta a=\frac{2\left(D_{1}^{*} D^{*} \theta_{1}^{2}+D_{2}^{*} D^{*}-D_{1}^{*} D_{2}^{*}-2 D_{1}^{*} D_{2}^{*} \theta_{1}-D_{1}^{*} D_{2}^{*} \theta_{1}^{2}\right)}{D^{*}\left(D_{2}^{*}-D_{1}^{*} \theta_{1}\right)}
$$

There are 13 pure modes within the context of Timoshenko beam theory, however the absence of interaction means that six of them from the first set coincide with six of them from the second set, giving seven unique pure modes (four pure mode I and three pure mode II). There are also two additional contributions to the mode I ERR $G_{I}$ : the ERR due to shearing, denoted by $G_{P}$; and the ERR due to interaction between the $\varphi_{\theta_{1}}$ mode crack tip opening force and the relative opening displacement due to shearing, and vice versa, denoted by $\Delta G_{\theta_{1} P}$. The ERRs are therefore

$$
\begin{gathered}
G=\frac{1}{2 b^{2}}\left(\frac{M_{1 B}^{2}}{D_{1}^{*}}+\frac{M_{2 B}^{2}}{D_{2}^{*}}-\frac{M_{B}^{2}}{D^{*}}+\frac{N_{1 B}^{2}}{A_{1}^{*}}+\frac{N_{2 B}^{2}}{A_{2}^{*}}-\frac{N_{B}^{2}}{A^{*}}-\frac{2 B_{1} M_{1 B} N_{1 B}}{B_{1}^{*}}\right. \\
\left.-\frac{2 B_{2} M_{2 B} N_{2 B}}{B_{2}^{*}}+\frac{2 B M_{B} N_{B}}{B^{*}}\right)+G_{P}+\alpha_{\theta_{1}} \Delta G_{\theta_{1} P} \\
G_{I}=\alpha_{\theta_{1}}^{2} G_{\theta_{1}}+G_{P}+\alpha_{\theta_{1}} \Delta G_{\theta_{1} P}
\end{gathered}
$$

where

$$
\begin{gathered}
G_{P}=\left(H_{1} P_{2 B}-H_{2} P_{1 B}\right)^{2} /\left[2 b^{2} H_{1} H_{2}\left(H_{1}+H_{2}\right)\right] \\
\Delta G_{\theta_{1} P}=\frac{1}{b^{2}}\left(\frac{P_{1 B}}{H_{1}}-\frac{P_{2 B}}{H_{2}}\right)\left[\frac{H_{1} H_{2}}{2\left(H_{1}+H_{2}\right)}\left(\frac{1}{D_{1}^{*}}+\frac{\theta_{1}^{2}}{D_{2}^{*}}-\frac{\left(1+\theta_{1}\right)^{2}}{D^{*}}\right)\right]^{1 / 2}
\end{gathered}
$$

The crack tip opening force $F_{n B}$, which is required to solve the governing equations from Timoshenko beam theory in Section 4.1, can now be derived. Since the $\varphi_{\beta_{i}}$ modes are characterized by zero opening force ahead of the crack tip, the crack tip opening force $F_{n B}$ is given by

$$
F_{n B}=\alpha_{\theta_{1}} F_{n B \theta_{1}}+F_{n B P}
$$

where $\alpha_{\theta_{1}}$ is known in terms of the crack tip forces from Eq. (91), $F_{n B \theta_{1}}$ is the crack tip opening force in mode $\varphi_{\theta_{1}}$ and $F_{n B P}$ is the crack tip opening force due to shearing. From Timoshenko beam theory, they are

$$
F_{n B \theta_{1}}=\left[\frac{H_{1} H_{2}}{2\left(H_{1}+H_{2}\right)}\left(\frac{1}{D_{1}^{*}}+\frac{\theta_{1}^{2}}{D_{2}^{*}}-\frac{\left(1+\theta_{1}\right)^{2}}{D^{*}}\right)\right]^{1 / 2} \quad, \quad F_{n B P}=\frac{H_{2} P_{1 B}-H_{1} P_{2 B}}{H_{1}+H_{2}}
$$

The governing equations in Section 4.1 can now be solved to find the crack tip forces and obtain the ERR partition. 


\subsection{Two sets of orthogonal pure modes}

For the symmetric case with Euler beams, for which the crack tip forces are given by Eqs. (80) and (81), the F modes arising from the displacement conditions (i.e. zero relative shearing when $P_{2} / P_{1}=\theta_{F}$, and zero relative opening displacement when $P_{2} / P_{1}=\beta_{F}^{\prime}$ ) can be presented algebraically. By substituting the displacements and crack tip forces for this symmetric case into Eqs. (32) and (44) and equating them to zero, the following F modes are obtained:

$$
\begin{gathered}
P_{2} / P_{1}=\theta_{F}=-B_{2}^{*}\left(2 B_{1}+h_{1} A_{1}\right) /\left[B_{1}^{*}\left(2 B_{2}-h_{2} A_{2}\right)\right] \quad, \quad \varphi_{\theta_{F}}=\left\{\begin{array}{ll}
1 & \theta_{F}
\end{array}\right\}^{T} \\
P_{2} / P_{1}=\beta_{F}^{\prime}=D_{2}^{*} / D_{1}^{*} \quad, \quad \varphi_{\beta_{F}^{\prime}}=\left\{\begin{array}{ll}
1 & \beta_{F}^{\prime}
\end{array}\right\}^{T}
\end{gathered}
$$

The F modes arising from the zero crack tip opening force when $P_{2} / P_{1}=\beta_{F}$ condition is too extensive to be presented here algebraically. However, for specific cases, a numerical value for $\beta_{F}$ can be calculated by enforcing orthogonality with $\theta_{F}$. The ERR can be written as

$$
G=\left\{\begin{array}{ll}
P_{1} & P_{2}
\end{array}\right\}[C]\left\{\begin{array}{ll}
P_{1} & P_{2}
\end{array}\right\}^{T}
$$

where $[C]$ is found by examining coefficients of $P_{1}$ and $P_{2}$ in Eq. (83) when Eqs. (80) and (81) have been substituted in. Therefore $\beta_{F}$ can be found by solving

$$
0=\left\{\begin{array}{ll}
1 & \beta_{F}
\end{array}\right\}[C]\left\{1 \quad \theta_{F}\right\}^{T}
$$

Similarly $\theta_{F}^{\prime}$ can be found by solving

$$
0=\left\{\begin{array}{ll}
1 & \theta_{F}^{\prime}
\end{array}\right\}[C]\left\{\begin{array}{ll}
1 & \beta_{F}^{\prime}
\end{array}\right\}^{T}
$$

which always gives

$$
\theta_{F}^{\prime}=-1
$$

If the above procedure is repeated for Timoshenko beams then as before, it is found that the $\varphi_{\theta_{F}^{\prime}}$ mode changes to coincide with the $\varphi_{\theta_{F}}$ mode, which is different to that obtained from Euler beam theory. Similarly the $\varphi_{\beta_{F}^{\prime}}$ mode coincides with the $\varphi_{\beta_{F}}$ mode, which is also different to that obtained from Euler beam theory. The expressions for the Timoshenko ' $F$ modes' are long and complex in their general form, so are not presented here. They are however easily derived for specific cases with numerical quantities. Furthermore, when the through-thickness shear effect is not excessively large, Eqs. (102) and (105) are good approximations. 


\subsection{Contacting fractures}

To find the point of first contact $x_{c}$ using Euler beam theory, again the two conditions given by Eqs. (46) must be satisfied. Solving these equations simultaneously for $P_{2} / P_{1}$ and $x_{c}$ and ignoring the obvious and unavailing solutions for the crack tips, gives

$$
P_{2} / P_{1}=D_{2}^{*} / D_{1}^{*}=\beta_{F}^{\prime}
$$

for all values of $x$. This implies simultaneous contact everywhere along the fracture for this value of $P_{2} / P_{1}$.

If $P_{2} / P_{1}$ is increased beyond $\beta_{F}^{\prime}$, the contact can either be at a point or distributed. In the same way as before for the isotropic case, point contact at $x=x_{p}$ is assumed, which is a reasonable assumption, and shown to satisfy the requirement that it prevents intersection between the upper and lower sublaminates for all values of $x$.

Two loads $P_{1 c}$ and $P_{2 c}$ are applied to the beam at $x=x_{p}$ and they cause point contact at this same location. The point contact force $P_{c}$ acts to prevent non-physical interpenetration. The net shear loads $P_{1}$ and $P_{2}$ acting on the beams are given by Eq. (48). Eq. (49) is the equation that must be satisfied to prevent intersection at $x=x_{p}$. Solving Eqs. (48) and (49) for $P_{1}, P_{2}$ and $P_{c}$ gives

$$
\begin{gathered}
P_{1}=A_{2} B_{1}^{*}\left(P_{1 c}+P_{2 c}\right) /\left(A_{2} B_{1}^{2}+A_{1} B_{2}^{2}-A_{1} A_{2} D_{1}-A_{1} A_{2} D_{2}\right) \\
P_{2}=A_{1} B_{2}^{*}\left(P_{1 c}+P_{2 c}\right) /\left(A_{2} B_{1}^{2}+A_{1} B_{2}^{2}-A_{1} A_{2} D_{1}-A_{1} A_{2} D_{2}\right) \\
P_{c}=\left[\left(A_{1} A_{2} D_{2}-A_{1} B_{2}^{2}\right) P_{1 c}-\left(A_{1} A_{2} D_{1}-A_{2} B_{1}^{2}\right) P_{2 c}\right] /\left(A_{2} B_{1}^{2}+A_{1} B_{2}^{2}-A_{1} A_{2} D_{1}-A_{1} A_{2} D_{2}\right)
\end{gathered}
$$

Substituting Eqs. (109) to (111) into $D_{o p}=w_{1}-w_{2}$ reveals that $D_{o p}=0$ for all values of $x$.

Therefore the requirements for physical contact behavior are satisfied by this solution, demonstrating that it is the correct one.

Using Timoshenko beam theory, at $P_{2} / P_{1}=\beta_{F}=\beta_{F}^{\prime}$ there is both zero opening force beyond the crack tip and zero relative opening displacement just behind. Therefore crack tip running contact occurs at $P_{2} / P_{1}=\beta_{F}$ and a pure mode II fracture is obtained. Since there is running contact, if the loading ratio $P_{2} / P_{1}$ is increased further then the crack tip remains closed as the contacting region grows. 


\section{Numerical investigations}

To verify the theory, a finite element method (FEM) simulation capability was developed based on the Euler and Timoshenko beam theories and 2D elasticity. Normal and shear point interface springs with the very high stiffness of $10^{14} \mathrm{~N} / \mathrm{m}$ were used to model perfectly bonded plies (14,26-29). Through convergence studies this value was found to be large enough to approach the behavior of a rigid interface, but not so high as to introduce excessive numerical error. The ERR partition was calculated using the virtual crack closure technique in conjunction with these interface springs $(14,26$ 29). A contact algorithm was also implemented to deal with any possible contact in loading.

Two clamped-clamped beam cases were investigated. The first case is an asymmetric, isotropic one, the data for which is given in Table 1.1. The second case is a symmetric laminated composite one. It has a quasi-isotropic lay-up with 16 plies. There is a delamination between the fourth and fifth plies, which gives a thickness ratio of $\gamma=3$. The data for this case is given in Table 1.2. The material properties are for a T300/976 graphite/epoxy ply (28).

One set of simulations, which used linear Timoshenko beam elements, is compared against the Euler beam theory. Very large out-of-plane shear moduli $G_{x z}=G_{13}=G_{23}=10^{16} \mathrm{~N} / \mathrm{m}$ were used to simulate Euler beam theory. As is the case for the spring stiffness, convergence studies were carried out and this value for $G_{x z}$ was found to be large enough to approach the behavior of Euler beams, but not so high as to introduce excessive numerical error. Two layers of elements were used to represent the beams with one on either side of the fracture. The elements were distributed uniformly. To avoid shear locking, reduced integration was applied. Use of linear Timoshenko beams correctly enforces continuity along the interface ahead of the crack tip.

Another set of simulations, which was the same as the first set but which instead used the normal outof-plane shear moduli (those given in Tables 1.1 and 1.2) and a shear correction factor of $\kappa^{2}=5 / 6$, is compared against the Timoshenko beam theory.

The final simulations used four-node quadrilateral (QUAD4) finite elements with the normal out-ofplane shear moduli. Layers of QUAD4 elements model the sub-laminates and they are also joined with very high stiffness normal and shear interface springs. In the composite case, a layer of QUAD4 elements was used for each individual ply. This was found to be necessary to obtain converged results. In the isotropic case, two and four layers of QUAD4 elements were needed in the top and bottom beams respectively for sufficient convergence. The elements were distributed uniformly along the length and thickness. The results from these simulations are compared against the Euler and Timoshenko theories and an averaged partition rule. 
The following sections present the results from these three sets of simulations for the two different cases. The only applied loads are $P_{1}$ and $P_{2} ; P_{1}$ was held constant at $1 \mathrm{~N}$ and $P_{2}$ was varied.

\subsection{Tests with clamped-clamped isotropic beams}

Results from the various analytical theories and numerical simulations of the isotropic clampedclamped beam are presented in Tables 1.3 and 1.4 and Figure 1.3. Plane stress is assumed in all analytical and numerical calculations. The ERR partition for the left crack tip is given. In Figure 1.3 and for every figure in this section, unfilled data markers indicate results from simulations with contact modeling and filled markers indicate results from simulations without. The results from the simulations using Timoshenko beam elements and the very large shear modulus are compared against the Euler beam partition theory. Excellent agreement is seen between the two sets of data. The two sets of pure modes are plainly visible where $G_{I} / G=0$ and $G_{I} / G=1$. The two methods are in agreement that point contact at the loading location and at the crack tips contact occur simultaneously at $P_{2}=8 \mathrm{~N}$; and that after first contact, both crack tips remain closed and the fracture is pure mode II.

The Timoshenko beam partition theory is compared with results from numerical simulations with the normal shear modulus. As expected, the $\varphi_{\theta_{\mathrm{F}}^{\prime}}$ and $\varphi_{\beta_{\mathrm{F}}^{\prime}}$ modes coincide with the $\varphi_{\theta_{\mathrm{F}}}$ and $\varphi_{\beta_{\mathrm{F}}}$ modes respectively. The numerical results with $800 \times 2$ elements very closely follow the analytical values. The results with $200 \times 2$ elements are in less good agreement. This demonstrates that the element size $\delta a$ needs to be very small otherwise $F_{n B \theta_{1}} \delta a$ is not negligible and a second set of pure modes is generated numerically. This is consistent with the discussion and observations in previous work (1013). As expected, crack tip running contact begins at the $\varphi_{\beta_{F}}$ mode. Crack tip running contact necessarily gives $G_{I} / G=0$. Beyond the $\varphi_{\beta_{F}}$ mode, the crack tips remain closed. The numerical simulations model this contact behavior very closely.

An 'averaged partition rule' has been tested in previous work (10-13) and has been found to generally give good agreement with the fracture mode partition from 2D elasticity for: (1) all thickness ratios, (2) all loading conditions, and (3) all material properties, including laminated composite. Particularly regarding material properties, there is some complex mechanical behavior in the case of even simple laminates like bimaterials (20). However despite this, the averaged rule can still provide a reasonable approximation. Readers are referred to these publications (10-13) for further details. Detailed papers by the authors on the topic of fractures on bimaterial interfaces are in preparation $(24,25)$. The averaged rule is as follows: 


$$
G_{I}=\alpha_{\theta_{1}}^{2} G_{\theta_{1}}+\alpha_{\theta_{1}} \alpha_{\beta_{1}} \Delta G_{\theta_{1} \beta_{1}} / 2+\alpha_{\theta_{1}} \alpha_{\beta_{2}} \Delta G_{\theta_{1} \beta_{2}} / 2+G_{P}+\alpha_{\theta_{1}} \Delta G_{\theta_{1} P}
$$

The effect of shearing is small in this case because the beam is relatively thin. Therefore the averaged fracture mode partition lies approximately midway between the Euler and Timoshenko curves. There is excellent agreement between this curve and the 2D FEM results. In addition to the above, it is once again seen that the $\varphi_{\theta_{F}}$ and $\varphi_{\beta_{F}}$ modes are still approximately the pure modes.

\subsection{Tests with clamped-clamped laminated composite beams}

The data is now presented for the clamped-clamped laminated composite beam. The plane-strain assumption was used in all these analytical and numerical calculations. Under this assumption, $A=A_{11}, B=B_{11}, D=D_{11}$ and $H=A_{55}$.

Since many of the observations are the same as for the isotropic case, they are not repeated. New observations are simply added. Tables 1.5 and 1.6 and Figure 1.4 present results from the various analytical theories and numerical simulations of the laminated composite clamped-clamped beam are presented in. There is excellent agreement between the Euler beam partition theory and the Euler numerical results. There is also excellent agreement between the Timoshenko beam theory and the Timoshenko numerical results.

In this composite case there is a much larger difference between the $\varphi_{\theta_{F}}$ and $\varphi_{\theta_{F}^{\prime}}$ modes than what was seen for the isotropic case (compare Tables 1.1 and 1.2). Having an Euler curve with substantially different $\varphi_{\theta_{F}}$ and $\varphi_{\theta_{F}^{\prime}}$ modes makes it substantially different to the Timoshenko curve. This large difference might therefore have strained the accuracy of the average partition approximation. Despite this possibility, the agreement observed between the averaged partition and the 2D FEM is excellent for the whole the range of $P_{2}$ simulated.

\section{Further discussion and conclusions}

Analytical theories have been developed for mixed-mode delamination in layered isotropic and laminated composite straight beam structures. Unlike the theories developed by the authors in Refs. (9-18) for mixed-mode cracks in layered isotropic and laminated composite DCBs, in these beam structures the internal forces at the crack tips are generally complex functions of remotely applied loads. It is not generally possible to obtain pure 'crack tip modes', i.e. modes which relate crack tip quantities, because these quantities cannot be set independently of each other. Instead some combinations of these modes can give pure mode I or II fractures. 
This work mainly focused on the most common practical cases of layered isotropic and laminated composite straight beam structures with shear forces applied at an arbitrary location in the delaminated region. For these beams, the ' $F$ modes' have been derived for each crack tip. The F modes give the ratios required between applied shear forces $P_{1}$ and $P_{2}$, to give pure fractures modes.

The theories have been developed based on the Euler and Timoshenko isotropic and laminated composite beam theories. Both theories have their own orthogonal $\varphi_{\theta_{F}}$ and $\varphi_{\beta_{F}}$ pure modes which are called the first set. They correspond to zero relative shearing displacement just behind the crack tip and zero crack tip opening force respectively. For the statically indeterminate beam structures examined in this paper, the first set of pure modes from Euler beam theory is generally different in value to the first set from Timoshenko beam theory. However when the through-thickness effect is small, the Euler pure modes may be a close approximation to the Timoshenko pure modes.

In Euler beam theory, there is a second set of orthogonal pure modes $\varphi_{\theta_{F}^{\prime}}$ and $\varphi_{\beta_{F}^{\prime}}$, which are different to the first set. They correspond to zero crack tip shearing force and zero relative opening displacement just behind the crack tip respectively. Within the context of Timoshenko beam theory, the $\varphi_{\theta_{F}^{\prime}}$ and $\varphi_{\beta_{F}^{\prime}}$ modes coincide with the $\varphi_{\theta_{F}}$ and $\varphi_{\beta_{F}}$ modes. Therefore the $\varphi_{\theta_{F}}$ and $\varphi_{\beta_{F}}$ modes form a complete basis for mixed mode partitions.

The Euler and Timoshenko beam theory mode partitions agree very well with the corresponding beam FEM predictions. The averaged partition approximation, which has been described in previous work by the authors (10-13), has been further tested. The approximation generally agrees very well with the 2D FEM results, even when the difference between the Euler and Timoshenko curves is substantial and the accuracy of the approximation might have become strained.

The developed theories will be a valuable analytical tool in many applications for example for analytical researchers to develop fracture propagation criteria; for design engineers to design high integrity structures and for numerical analysts to benchmark their simulations, etc. These theories have also been extended to isotropic and composite axisymmetric plates, curved beams and shells; they will be reported in a future paper.

\section{References}

1. Williams, J.G. On the calculation of energy release rates for cracked laminates. Int. J. Fract. Mech. 1988,36, 101-119.

2. Schapery, R.A.; Davidson, B.D. Prediction of energy release rate for mixed-mode delamination using classical plate theory. Appl. Mech. Rev. 1990,43, S281-287. 
3. Suo, Z. Delamination specimens for orthotropic materials. J. Appl. Mech. 1990,56, 627-634.

4. Suo, Z.; Hutchinson, J.W. Interface crack between two elastic layers. Int. J. Fract. Mech. 1990,43, 1-18.

5. Hutchinson, J.W.; Suo, Z. Mixed mode cracking in layered materials. Adv. Appl. Mech. 1992,29, 63-191.

6. Zou, Z.; Reid, S.R.; Li, S.; Soden, P.D. General expressions for energy release rates for delamination in composite laminates. Proc. R. Soc. A 2002,458, 645-667.

7. Bruno, D.; Greco, F. Mixed mode delamination in plates: A refined approach. Int. J. Solids Struct. 2001,38, 9149-9177.

8. Luo, Q.; Tong, L. Calculation of energy release rates for cohesive and interlaminar delamination based on the classical beam-adhesive model. J. Compos. Mater. 2009,43, 331-348.

9. Wang, S.; Harvey, C.M. Mixed mode partition in one-dimensional fracture. J. Key Eng. Mater. 2011,462-463, 616-621. Also, a plenary lecture in the $8^{\text {th }}$ International Conference on Fracture and Strength of Solids (FEOFS 2010), 7-9 ${ }^{\text {th }}$ June 2010, Kuala Lumpur, Malaysia.

10. Wang, S.; Harvey, C.M. A theory of one-dimensional fracture. Compos. Struct. 2012,94, 758767. Also, a plenary lecture in the $16^{\text {th }}$ International Conference on Composite Structures (ICCS16), 28-30 ${ }^{\text {th }}$ June 2011, Porto, Portugal.

11. Harvey, C.M.; Wang, S. Experimental assessment of mixed-mode partition theories. Compos. Struct. 2012,94, 2057-2067.

12. Wang, S.; Harvey, C.M. Mixed mode partition theories for one dimensional fracture. Eng. Fract. Mech. 2012,79, 329-352.

13. Harvey, C.M.; Wang, S. Mixed-mode partition theories for one-dimensional delamination in laminated composite beams. Eng. Fract. Mech. 2012,96, 737-759.

14. Harvey, C.M. Mixed-mode partition theories for one-dimensional fracture. PhD Dissertation, Loughborough University, UK, 2012.

15. Wang, S.; Guan, L. On fracture mode partition theories. Comp. Mater. Sci. 2012,52, 240-245.

16. Wang, S.; Harvey, C.M.; Guan, L. Partition of mixed modes in layered isotropic double cantilever beams with non-rigid cohesive interfaces. Eng. Fract. Mech. 2013,111, 1-25.

17. Harvey, C.M.; Wood, J.D.; Wang, S.; Watson, A. A novel method for the partition of mixedmode fractures in 2D elastic laminated unidirectional composite beams. Compos. Struct. 2014,116, 589-594.

18. Harvey, C.M.; Eplett, M.R.; Wang, S. Experimental assessment of mixed-mode partition theories for generally laminated composite beams. Compos. Struct. 2015,124, 10-18. 
19. Charalambides, M.; Kinloch, A.J.; Wang, W.; Williams, J.G. On the analysis of mixed-mode failure. Int. J. Fract. Mech. 1992,54, 269-291.

20. Hashemi, S.; Kinloch, A.J.; Williams, G. Mixed-mode fracture in fiber-polymer composite laminates. In Composite materials: Fatigue and fracture, ASTM STP 1110; O’Brien, T.K., Ed.; American Society for Testing and Materials: Philadelphia, PA, 1991; Vol. 3; p. 143-168.

21. Davidson, B.D.; Gharibian, S.J.; Yu, L. Evaluation of energy release rate-based approaches for predicting delamination growth in laminated composites. Int. J. Fract. Mech. 2000,105, 343-365.

22. Davidson, B.D.; Bialaszewski, R.D.; Sainath, S.S. A non-classical, energy release rate based approach for predicting delamination growth in graphite reinforced laminated polymeric composites. Compos. Sci. Technol. 2006,66, 1479-1496.

23. Conroy, M.; Sørensen, B.F.; Ivankovic, A. Combined numerical and experimental investigation of mode-mixity in beam like geometries. Proceeding of the 7th International Conference on Fracture of Polymers, Composites and Adhesives, Les Diablerets, Switzerland, September 14-18, 2014.

24. Harvey, C.M.; Wood, J.D.; Wang, S. Brittle interfacial cracking between two dissimilar elastic layers. Part 1-Analytical development. Compos. Struct. 2015 (In preparation).

25. Harvey, C.M.; Wood, J.D.; Wang, S. Brittle interfacial cracking between two dissimilar elastic layers. Part 2-Numerical validation. Compos. Struct. 2015 (In preparation).

26. Harvey, C.M.; Wang, S. Modelling of delamination propagation in composite laminated beam structures. AIP Conf. Proc. 2012,1504, 1146-1149.

27. Harvey, C.M.; Wang, S. Numerical and analytical study of delamination in composite laminates, Int. J. Eng. Syst. Model. Sim. 2012,4, 120-137.

28. Wang, S.; Zhang, Y. Buckling, post-buckling and delamination propagation in debonded composite laminates. Part 2: Numerical applications. Compos. Struct. 2009,88, 131-146.

29. Kutlu, Z.; Chang, F.K. Composite panels containing multiple through-the-width delaminations and subjected to compression. Part II: Experiments and verification. Compos. Struct. 1995,31, 297-314. 
Table 1.1: Data for numerical simulations of a clamped-clamped isotropic beam

\begin{tabular}{cc} 
Elastic modulus, $E$ & $70 \mathrm{GPa}$ \\
Shear modulus, $G_{x z}$ & $26 \mathrm{GPa}$ \\
Poisson's ratio, $v_{x z}$ & 0.35 \\
Beam thicknesses, $h_{1}$ and $h_{2}$ & $1 \mathrm{~mm}$ and $2 \mathrm{~mm}$ \\
Intact lengths of beam, $L_{1}$ and $L_{2}$ & $10 \mathrm{~mm}$ and $25 \mathrm{~mm}$ \\
Length of fracture, $a$ & $65 \mathrm{~mm}$ \\
Width of beam, $b$ & $10 \mathrm{~mm}$ \\
Loading location, $x_{p}$ & $20 \mathrm{~mm}$ \\
Euler pure modes $\theta_{F}, \beta_{F}, \theta_{F}{ }^{\prime}$ and $\beta_{F}{ }^{\prime}$ & $-3.92,2.81,-1$ and 8 \\
Timoshenko pure modes $\theta_{F}$ and $\beta_{F}$ & -3.84 and 2.75 \\
\hline
\end{tabular}

Table 1.2: Data for numerical simulations of a clamped-clamped laminated composite beam

\begin{tabular}{|c|c|}
\hline Ply longitudinal modulus, $E_{11}$ & $139.3 \mathrm{GPa}$ \\
\hline Ply transverse modulus, $E_{22}$ & $9.72 \mathrm{GPa}$ \\
\hline Out-of-plane modulus, $E_{33}$ & $9.72 \mathrm{GPa}$ \\
\hline In-plane shear modulus, $G_{12}$ & $5.58 \mathrm{GPa}$ \\
\hline Out-of-plane shear moduli, $G_{13}$ & $5.58 \mathrm{GPa}$ \\
\hline Out-of-plane shear moduli, $G_{23}$ & $3.45 \mathrm{GPa}$ \\
\hline In-plane Poisson's ratio, $v_{12}$ & 0.29 \\
\hline Out-of-plane Poisson's ratio, $v_{13}$ & 0.29 \\
\hline Out-of-plane Poisson’s ratio, $v_{23}$ & 0.4 \\
\hline Ply thickness, $t_{p}$ & $0.125 \mathrm{~mm}$ \\
\hline Sub-laminate lay-up 1 (top) & $90 /-45 / 0 / 45$ \\
\hline Sub-laminate lay-up 2 (bottom) & $(45 / 0 /-45 / 90)_{2} / 90 /-45 / 0 / 45$ \\
\hline Laminate thicknesses, $h_{1}$ and $h_{2}$ & $0.5 \mathrm{~mm}$ and $1.5 \mathrm{~mm}$ \\
\hline Intact lengths of beam, $L_{1}=L_{2}$ & $25 \mathrm{~mm}$ \\
\hline Length of fracture, $a$ & $50 \mathrm{~mm}$ \\
\hline Width of beam, $b$ & $10 \mathrm{~mm}$ \\
\hline Loading location, $x_{p}$ & $25 \mathrm{~mm}$ \\
\hline er pure modes $\theta_{F}, \beta_{F}, \theta_{F}{ }^{\prime}$ and $\beta_{F}{ }^{\prime}$ & $-26.45,4.98,-1$ and 66.90 \\
\hline imoshenko pure modes $\theta_{F}$ & -23.20 and 4.74 \\
\hline
\end{tabular}


Table 1.3: Comparison between various theories for clamped-clamped isotropic beam energy release rate partitions with varying $P_{2}$ and $P_{1}=1 \mathrm{~N}$.

\begin{tabular}{cccccccc}
\hline & \multicolumn{7}{c}{$G_{I} / G(\%)$} \\
\cline { 2 - 8 }$P_{2}(\mathrm{~N})$ & $\begin{array}{c}\text { Analytical } \\
\text { Euler }\end{array}$ & $\begin{array}{c}\text { Numerical } \\
\text { Euler } \\
(100 \times 2 \\
\text { Timo. } \\
\text { beams })\end{array}$ & $\begin{array}{c}\text { Analytical } \\
\text { Timo. }\end{array}$ & $\begin{array}{c}\text { Numerical } \\
\text { Timo. } \\
(800 \times 2 \\
\text { Timo. } \\
\text { beams })\end{array}$ & $\begin{array}{c}\text { Numerical } \\
\text { Timo. } \\
(200 \times 2 \\
\text { Timo. } \\
\text { beams })\end{array}$ & $\begin{array}{c}\text { Averaged } \\
\text { Analytical } \\
\text { (Euler \& } \\
\text { Timo.) }\end{array}$ & $\begin{array}{c}\text { 2D FEM } \\
(400 \times 6 \\
\text { QUAD4s) }\end{array}$ \\
\hline-10 & 70.30 & 70.30 & 88.54 & 87.39 & 84.07 & 79.94 & 80.23 \\
-8 & 77.28 & 77.27 & 92.34 & 91.38 & 88.62 & 85.15 & 85.05 \\
-6 & 86.94 & 86.93 & 96.73 & 96.09 & 94.27 & 91.92 & 91.28 \\
-4 & 99.46 & 99.45 & 99.97 & 99.92 & 99.77 & 99.49 & 98.13 \\
-2 & 107.63 & 107.62 & 92.34 & 93.60 & 96.40 & 99.58 & 97.69 \\
0 & 76.34 & 76.34 & 48.13 & 51.43 & 56.70 & 61.84 & 60.69 \\
2 & 13.35 & 13.36 & 2.92 & 4.24 & 6.15 & 7.78 & 8.07 \\
4 & -7.25 & -7.23 & 4.36 & 3.05 & 0.99 & -0.87 & 0.91 \\
6 & -5.45 & -5.44 & 16.42 & 14.24 & 10.38 & 6.64 & 9.10 \\
8 & 0.00 & 0.01 & 26.16 & 23.76 & 19.15 & 14.47 & 17.14 \\
10 & 5.01 & 5.02 & 33.09 & 30.65 & 25.70 & 20.54 & 23.25 \\
\hline
\end{tabular}

Table 1.4: Comparison between various theories for clamped-clamped isotropic beam contact behavior with varying $P_{2}$ and $P_{1}=1 \mathrm{~N}$.

\begin{tabular}{|c|c|c|c|c|}
\hline & \multicolumn{2}{|c|}{ First contact } & \multicolumn{2}{|c|}{ After first contact } \\
\hline & $P_{2}(\mathrm{~N})$ & $G_{I} / G(\%)$ & $P_{2}(\mathrm{~N})$ & $G_{I} / G(\%)$ \\
\hline $\begin{array}{l}\text { Analytical } \\
\text { Euler }\end{array}$ & 8 & 0 & 10 & 0 \\
\hline $\begin{array}{l}\text { Numerical Euler } \\
(100 \times 2 \text { Timo. } \\
\text { beams })\end{array}$ & 7.99 & 0 & 0 & 0 \\
\hline $\begin{array}{l}\text { Analytical } \\
\text { Timo. }\end{array}$ & 2.75 & 0 & 10 & 0 \\
\hline $\begin{array}{l}\text { Numerical Timo } \\
\text { (800×2 Timo } \\
\text { beams })\end{array}$ & 3.06 & 0 & 10 & 0 \\
\hline $\begin{array}{l}\text { Numerical Timo } \\
\text { (200×2 Timo } \\
\text { beams) }\end{array}$ & 3.67 & 0 & 10 & 0 \\
\hline $\begin{array}{c}\text { Averaged } \\
\text { Analytical (Euler } \\
\text { \& Timo.) }\end{array}$ & 4.33 & 0 & 10 & 0 \\
\hline $\begin{array}{l}\text { 2D FEM ( } 400 \times 6 \\
\text { QUAD4s) }\end{array}$ & 3.52 & 0 & 10 & 0 \\
\hline
\end{tabular}


Table 1.5: Comparison between various theories for clamped-clamped laminated composite beam energy release rate partitions with varying $P_{2}$ and $P_{1}=1 \mathrm{~N}$.

\begin{tabular}{cccccccc}
\hline & \multicolumn{7}{c}{$G_{I} / G(\%)$} \\
\cline { 2 - 8 }$P_{2}(\mathrm{~N})$ & $\begin{array}{c}\text { Numerical } \\
\text { Analytical } \\
\text { Euler }\end{array}$ & $\begin{array}{c}\text { Euler } \\
(100 \times 2 \\
\text { Timo. } \\
\text { beams })\end{array}$ & $\begin{array}{c}\text { Analytical } \\
\text { Timo. }\end{array}$ & $\begin{array}{c}\text { Numerical } \\
\text { Timo. } \\
(800 \times 2 \\
\text { Timo. } \\
\text { beams })\end{array}$ & $\begin{array}{c}\text { Numerical } \\
\text { Timo. } \\
(200 \times 2 \\
\text { Timo. } \\
\text { beams })\end{array}$ & $\begin{array}{c}\text { Averaged } \\
\text { Analytical } \\
\text { (Euler \& } \\
\text { Timo.) }\end{array}$ & $\begin{array}{c}\text { 2D FEM } \\
(200 \times 16 \\
\text { QUAD4s) }\end{array}$ \\
\hline-10 & 145.33 & 145.33 & 87.57 & 89.20 & 98.46 & 113.51 & 101.99 \\
-8 & 147.63 & 147.62 & 79.89 & 82.13 & 93.05 & 111.45 & 100.40 \\
-6 & 145.01 & 145.00 & 68.83 & 71.93 & 84.24 & 105.37 & 95.65 \\
-4 & 134.61 & 134.60 & 54.04 & 58.15 & 71.19 & 93.47 & 86.17 \\
-2 & 114.10 & 114.09 & 36.51 & 41.43 & 53.97 & 74.79 & 70.87 \\
0 & 83.82 & 83.81 & 19.27 & 24.13 & 34.51 & 50.90 & 50.73 \\
2 & 48.24 & 48.24 & 6.41 & 9.90 & 16.58 & 26.46 & 29.71 \\
4 & 14.30 & 14.30 & 0.47 & 1.65 & 3.83 & 6.72 & 12.78 \\
6 & -12.60 & -12.61 & 0.98 & -0.24 & -2.39 & -5.75 & 2.50 \\
8 & -30.77 & -30.77 & 5.66 & 2.59 & -3.12 & -11.51 & -1.66 \\
10 & -41.30 & -41.29 & 12.21 & 7.99 & -0.36 & -12.56 & -1.69 \\
\hline
\end{tabular}

Table 1.6: Comparison between various theories for clamped-clamped laminated composite beam contact behavior with varying $P_{2}$ and $P_{1}=1 \mathrm{~N}$.

\begin{tabular}{ccccc}
\hline & \multicolumn{2}{c}{ First contact } & \multicolumn{2}{c}{ After first contact } \\
\cline { 2 - 5 } & $P_{2}(\mathrm{~N})$ & $G_{I} / G(\%)$ & $P_{2}(\mathrm{~N})$ & $G_{I} / G(\%)$ \\
\hline $\begin{array}{c}\text { Analytical } \\
\text { Euler }\end{array}$ & 66.90 & 0 & 100 & 0 \\
$\begin{array}{c}\text { Numerical Euler } \\
(100 \times 2 \text { Timo. } \\
\text { beams })\end{array}$ & 66.77 & 0 & 100 & 0 \\
$\begin{array}{c}\text { Analytical } \\
\text { Timo. }\end{array}$ & 4.74 & 0 & 100 & 0 \\
$\begin{array}{c}\text { Numerical Timo. } \\
\text { (800×2 Timo. } \\
\text { beams) }\end{array}$ & 6.38 & 0 & 100 & 0 \\
$\begin{array}{c}\text { Numerical Timo. } \\
\text { (200×2 Timo. } \\
\text { beams) }\end{array}$ & 10.18 & 0 & 100 & 0 \\
$\begin{array}{c}\text { Averaged } \\
\text { Analytical (Euler } \\
\text { \& Timo.) } \\
\text { 2D FEM } \\
\text { (200×16 }\end{array}$ & 18.23 & 0 & 100 & 0 \\
QUAD4s) & 11.60 & 0 & 100 & 0 \\
\hline
\end{tabular}




\section{Figure captions}

Figure 1.1: A clamped-clamped beam with a fracture and its loading conditions. (a) General description. (b) Force diagram of each beam.

Figure 1.2: Details of the crack influence region $\Delta a$ ahead of the left crack tip.

Figure 1.3: Comparison between various theories for clamped-clamped isotropic beam energy release rate partitions with varying $P_{2}$ and $P_{1}=1 \mathrm{~N}$.

Figure 1.4: Comparison between various theories for clamped-clamped laminated composite beam energy release rate partitions with varying $P_{2}$ and $P_{1}=1 \mathrm{~N}$. 


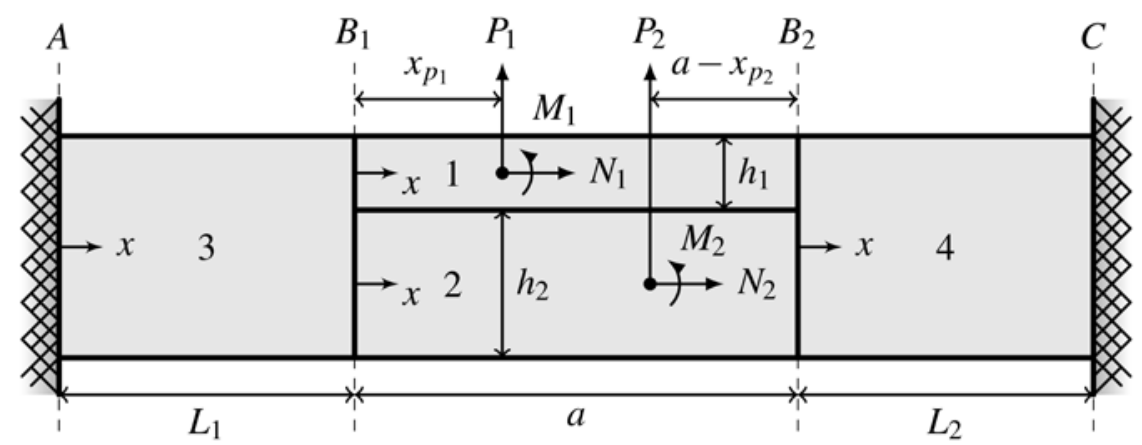

(a)

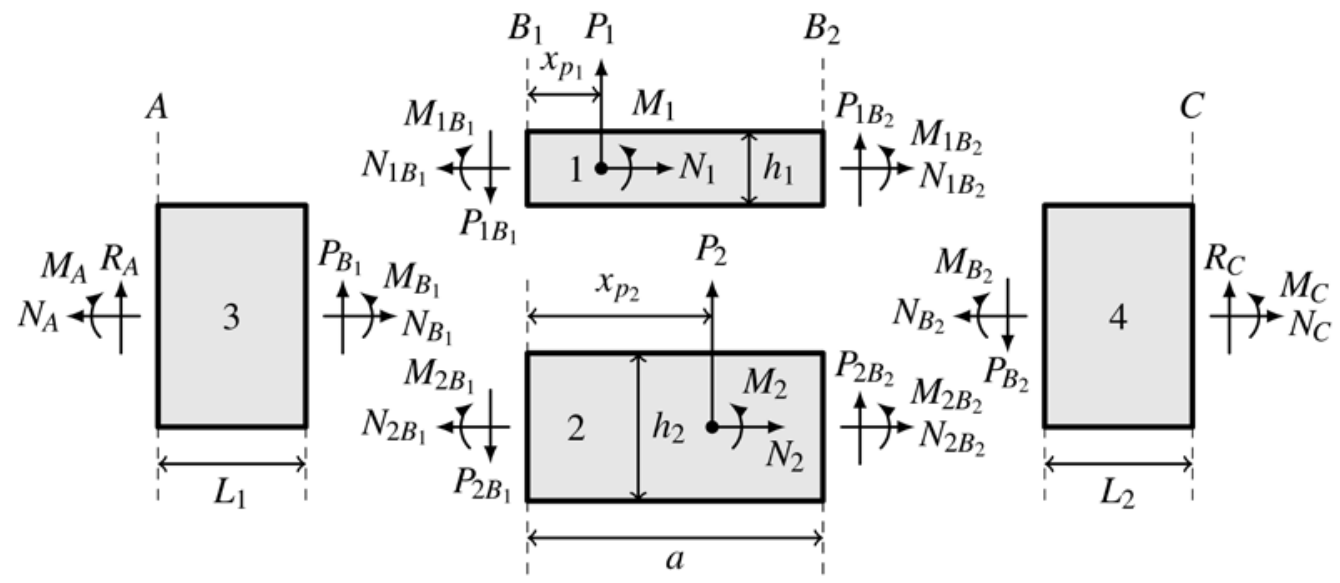

(b)

Figure 1.1: A clamped-clamped beam with a fracture and its loading conditions. (a) General description. (b) Force diagram of each beam. 


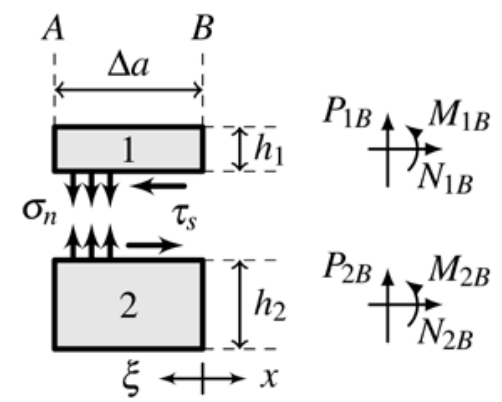

Figure 1.2: Details of the crack influence region $\Delta a$ ahead of the left crack tip. 


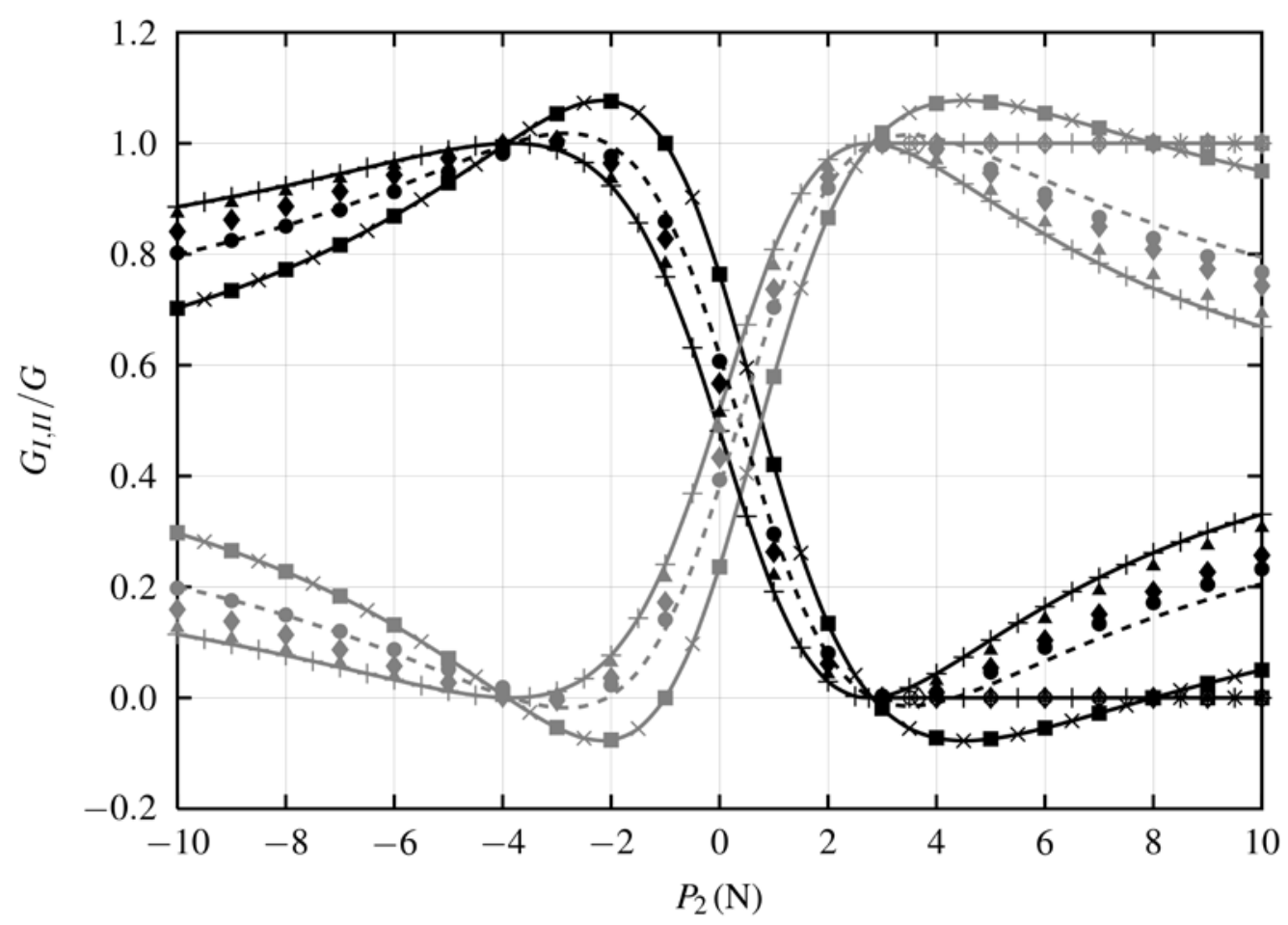

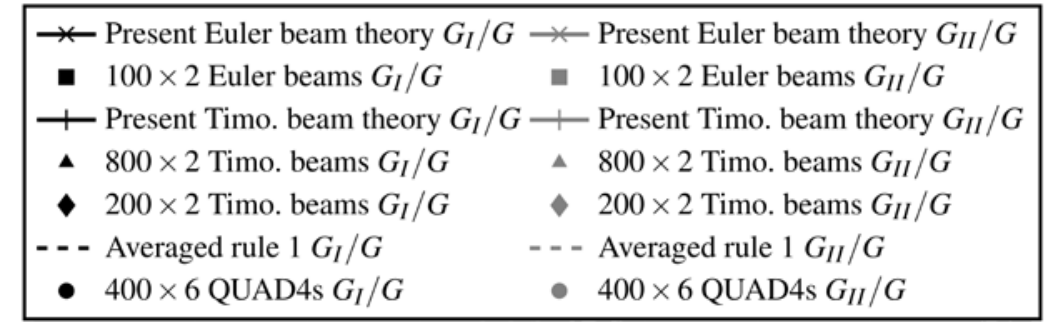

Figure 1.3: Comparison between various theories for clamped-clamped isotropic beam energy release rate partitions with varying $P_{2}$ and $P_{1}=1 \mathrm{~N}$. 


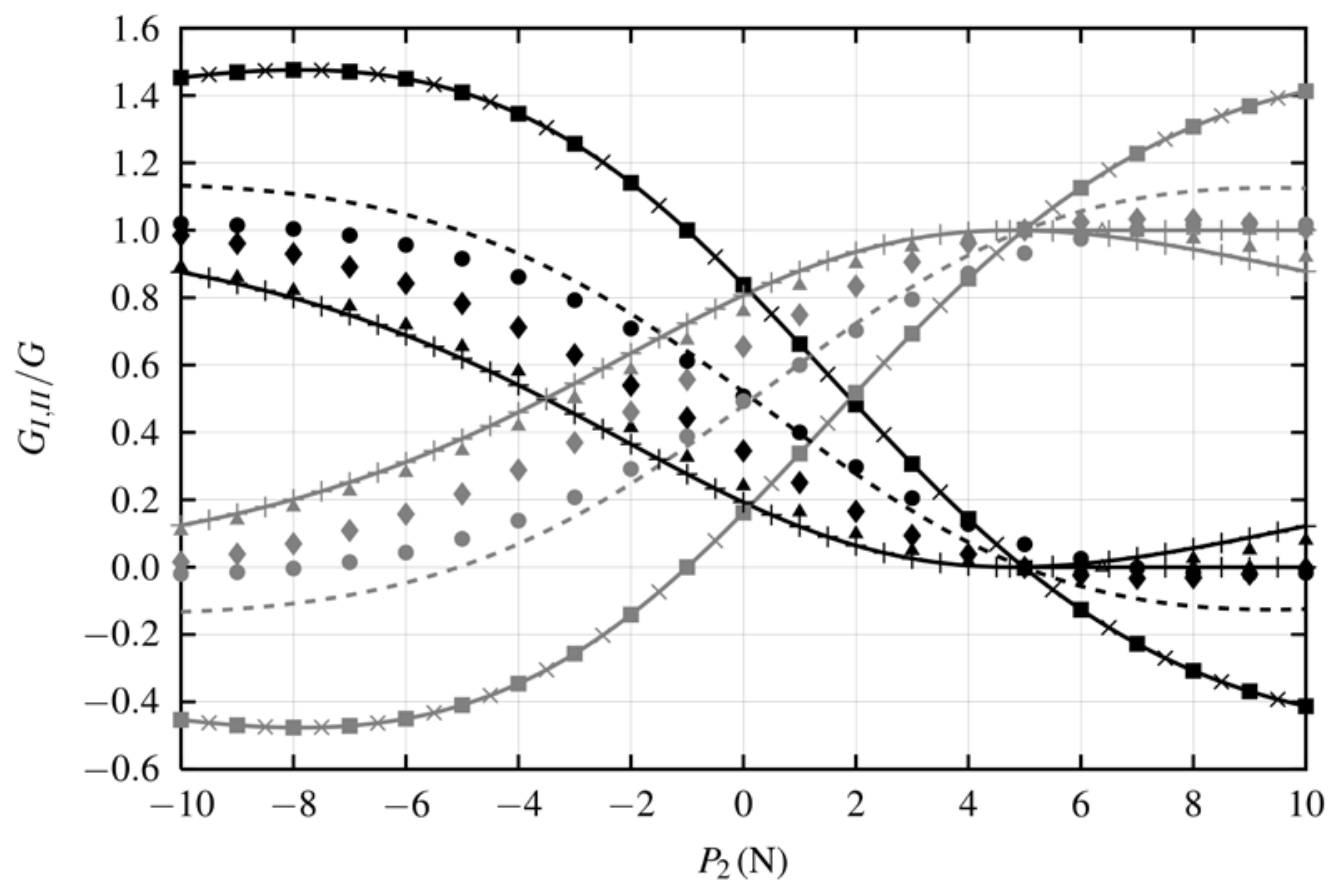

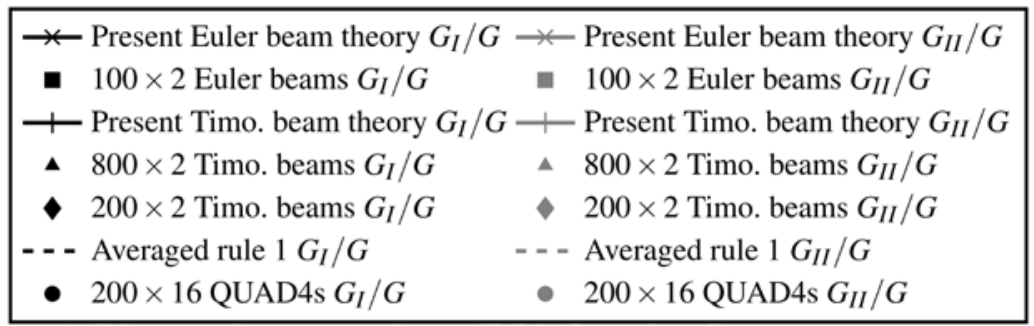

Figure 1.4: Comparison between various theories for clamped-clamped laminated composite beam energy release rate partitions with varying $P_{2}$ and $P_{1}=1 \mathrm{~N}$. 